\title{
Harnack estimates for weak supersolutions to nonlinear degenerate parabolic equations
}

\author{
TUOMO KUUSI
}

\begin{abstract}
In this work we prove both local and global Harnack estimates for weak supersolutions to second order nonlinear degenerate parabolic partial differential equations in divergence form. We reduce the proof to an analysis of so-called hot and cold alternatives, and use the expansion of positivity together with a parabolic type of covering argument. Our proof uses only the properties of weak supersolutions. In particular, no comparison to weak solutions is needed.
\end{abstract}

Mathematics Subject Classification (2000): 35K65(primary); 35K10, 35B45 (secondary).

\section{Introduction}

Harnack estimates play a central role in the regularity theory of partial differential equations. In this work we prove the parabolic weak Harnack estimate for weak supersolutions of the equation

$$
\frac{\partial u}{\partial t}-\operatorname{div}(\mathcal{A}(x, t, u, \nabla u))=0
$$

in $\mathbb{R}^{n} \times \mathbb{R}$, where the function $\mathcal{A}$ has a growth of order $p, p>2$, with respect to the norm of the gradient. In addition, it is assumed to be a Caratheodory function. These conditions and the definition of weak supersolutions are described in detail in Section 2. Our proof uses only measure theoretical arguments and no comparison to weak solutions is needed.

The problem has a long history in the field of nonlinear degenerate diffusion equations. The celebrated result of Moser in [24], see also [25] and [26], was the Harnack inequality for weak solutions to linear parabolic equations with bounded measurable coefficients. Later Aronson and Serrin [3], Ivanov [18], Kurihara [21] and Trudinger [27] generalized independently Moser's result for the quasilinear case. Trudinger explicitly pointed out that the Harnack inequality for weak solutions is a consequence of the weak Harnack estimate for weak supersolutions and 
reverse Hölder's inequality for weak subsolutions. He also stated that it is an open problem what are the suitable generalizations of Harnack estimates for equations with growth of order $p$ instead of quadratic growth [27, page 206].

Recently DiBenedetto, Gianazza and Vespri made a breakthrough by proving the intrinsic Harnack inequality for weak solutions to the equation with growth of order $p$ and bounded measurable coefficients, see $[9,10]$. In their proof they use neither Hölder continuity of solutions nor comparison to any fundamental solution. They also pay attention to the stability of constants as $p \rightarrow 2$. This generalizes Moser's result. Their proof uses extensively De Giorgi's estimates [7].

In this work we prove the weak Harnack estimate. As a consequence we also obtain the Harnack inequality. Since we operate with weak supersolutions, our technique differs from the one in [9]. Our emphasis is on the different roles of superand subsolutions, which resembles the original approach of Moser and Trudinger. We show that for the equations with growth of order $p$, the local weak Harnack estimate takes the following form.

Theorem 1.1. Let $u$ be a non-negative weak supersolution in $B\left(x_{0}, 8 R_{0}\right) \times\left(t_{0}, t_{0}+\right.$ $\left.T_{0}\right)$. Then there exist constants $C_{i}=C_{i}(n, p$, structure of $\mathcal{A}), i=1,2$, such that for almost every $t_{0}<t_{1}<t_{0}+T_{0}$, we have

$$
f_{B\left(x_{0}, R_{0}\right)} u\left(x, t_{1}\right) d x \leq\left(\frac{C_{1} R_{0}^{p}}{T_{0}+t_{0}-t_{1}}\right)^{1 /(p-2)}+C_{2} \underset{Q}{\operatorname{essinf} u,}
$$

where $Q=B\left(x_{0}, 4 R_{0}\right) \times\left(t_{1}+T / 2, t_{1}+T\right)$ and

$$
T=\min \left\{T_{0}+t_{0}-t_{1}, C_{1} R_{0}^{p}\left(f_{B\left(x_{0}, R_{0}\right)} u\left(x, t_{1}\right) d x\right)^{2-p}\right\} .
$$

Note that the estimate is intrinsic in sense that the waiting time to take the essential infimum on the right hand side depends on the solution itself. In the global case a stronger result holds.

Theorem 1.2. Let $u$ be a non-negative weak supersolution to (1.1) in $\mathbb{R}^{n} \times\left(0, T_{0}\right)$. Then there exists a constant $C=C(n, p$, structure of $\mathcal{A})$ such that for almost every $0<t_{0}<T_{0}$, every $x_{0} \in \mathbb{R}^{n}, R>0$ and $0<T<T_{0}-t_{0}$ we have

$$
f_{B\left(x_{0}, R\right)} u\left(x, t_{0}\right) d x \leq\left(\frac{C R^{p}}{T}\right)^{1 /(p-2)}+C\left(\frac{T}{R^{p}}\right)^{n / p} \underset{Q}{\operatorname{essinf}} u^{\lambda / p},
$$

where $\lambda=n(p-2)+p$ and $Q=B\left(x_{0}, 2 R\right) \times\left(t_{0}+T / 2, t_{0}+T\right)$.

We begin our proof of the local weak Harnack estimate by showing a Caccioppoli type estimate. For transparency of the work, we present all the needed results arising from the structure of the equation while showing this estimate. 
Next we show that supersolutions have a property called expansion of positivity. Although our method to show this differs from the one used in [9], the main point is similar. The contribution here is that we show how to establish the estimate using only the explicit properties of weak supersolutions. A byproduct of our method is that the stability of the constants as $p \rightarrow 2$ follows directly. By stability we mean that if $2<p<p_{0}$, then the constants may be chosen so that they depend only on $p_{0}$.

The core of our proof consists of two lemmas considering so-called hot and cold alternatives. We study non-negative weak supersolutions to (1.1) in the spacetime cylinder $B \times(0, T)$, where $B$ is a ball. We denote the positive initial mass in a ball $B^{\prime} \subset B$ by $M$. We show that for instants before $T$, the values of the supersolutions are essentially bounded below by a positive uniform constant depending only on the structure of the equation and $M$. This, however, realizes after a certain waiting time. The first case is that the domain is hot. By this we mean that the supersolution attains large values compared to $M$ in a relatively large set. This can happen, for instance, if the lateral boundary values are large. The high oscillation of the supersolution then hides the information about the initial mass. We overcome the difficulty with a refined Krylov-Safonov covering argument [20] together with the expansion of positivity. The version of the covering argument we prove here may be interesting as such. The argument is a parabolic counterpart of the argument of DiBenedetto and Trudinger in [13] for quasiminimizers. The presence of the waiting time makes the generalization rather delicate.

The second alternative is that the domain is cold. This means that the essential supremum of spatial integrals over the larger ball of a small power of the supersolution is bounded by a constant independent of $M$. This leads to a uniform estimate on the $L^{q}$-norm of the supersolution for some large $q$. This we establish by using Moser's iteration technique. The uniform estimate for the $L^{p-1}$-norm of the gradient then follows. These estimates, together with the expansion of positivity, imply the desired result provided that the initial mass $M$ is large enough.

As far as we know, the principal idea of the hot and cold alternatives is new. One of the main technical challenges of the work arises from the fact that the measure estimates obtained from the Krylov-Safonov covering argument are realized after a certain waiting time.

Theorems 1.1 and 1.2 are consequences of lemmas on the hot and cold alternatives, and a scaling argument. The constants $C_{1}$ and $C_{2}$ in Theorem 1.1 and $C$ in Theorem 1.2 are stable as $p \rightarrow 2$.

The global Harnack estimate, Theorem 1.2, is of the same type that Aronson and Caffarelli [2] proved for weak solutions to the porous medium equation. The corresponding result for a more general class of porous medium equations is due to Dahlberg and Kenig [5]. A good overview of techniques in [5] can be found in recent monograph [6] by Daskalopoulos and Kenig. See also monographs [29] and [30] by Vazquez. 
For weak solutions to the evolutionary $p$-Laplace equation

$$
\frac{\partial u}{\partial t}-\operatorname{div}\left(|\nabla u|^{p-2} \nabla u\right)=0
$$

Theorem 1.2 was proved by DiBenedetto and Herrero [11]. Choe and Lee [4] generalized it to an equation with a bounded measurable symmetric coefficient matrix depending only on the spatial variables by applying the method developed in [5]. The methods used in both [2] and [11] rely on the existence of a self similar solution, and, the techniques in [5] and [4] use the special symmetry of the weak solution. It is not clear how to generalize such methods to more general equations.

Our results apply to the equation

$$
\frac{\partial u}{\partial t}-\sum_{i=1}^{n} \frac{\partial}{\partial x_{i}}\left(\sum_{j=1}^{n} a_{i j}(x, t, u, \nabla u)\left|\frac{\partial u}{\partial x_{j}}\right|^{p-2} \frac{\partial u}{\partial x_{j}}\right)=0,
$$

where $p>2, a_{i j}(\cdot, \cdot, u, \xi)$ is a bounded measurable function for every $(u, \xi)$, continuous with respect to $u$ and $\xi$ for almost every $(x, t)$ and

$$
\mathcal{A}_{0}|\xi|^{2} \leq \sum_{i, j=1}^{n} a_{i j}(x, t, u, \xi) \xi_{i} \xi_{j} \leq \mathcal{A}_{1}|\xi|^{2}, \quad 0<\mathcal{A}_{0}<\mathcal{A}_{1}<\infty
$$

for almost every $(x, t)$ in $\mathbb{R}^{n} \times \mathbb{R}$ and every $(u, \xi)$ in $\mathbb{R} \times \mathbb{R}^{n}$. The particular case with the identity matrix $\left(a_{i j}\right)$ was studied by Lions in [22].

Weak supersolutions form an important class of functions, especially, in the analysis of the potential theoretical aspects of nonlinear partial differential equations in divergence form. Weak supersolutions relate in a natural way to weak solutions of the equation involving finite non-negative Radon measures on the right hand side of (1.1). For further discussion in this field, see the work of Acerbi and Mingione [1], and the references therein. In the elliptic theory, the weak Harnack estimate is one of the fundamental estimates - especially in the analysis of the equation with a non-negative measure on the right hand side. It is likely that the parabolic version of it will have a similar role. For further discussion on the potential theoretical aspects, see [17] by Heinonen, Kilpeläinen and Martio.

\subsection{Notation}

Our notation is standard. We denote the ball in $\mathbb{R}^{n}$ with the radius $R$ and center $x$ as $B(x, R)$. A space-time cylinder $\Omega \times\left(t_{1}, t_{2}\right)$ in $\mathbb{R}^{n} \times \mathbb{R}$ we call $\Omega_{t_{1}, t_{2}}$. The Lebesgue measure of the set $\Omega$ will be denoted by $|\Omega|$. By the notation $\Omega^{\prime} \Subset \Omega$ we mean that $\Omega^{\prime}$ belongs to $\Omega$ compactly. By the parabolic boundary of the set $\Omega_{t_{1}, t_{2}}$ we mean

$$
\partial_{p} \Omega_{t_{1}, t_{2}}=\left(\partial \Omega_{t_{1}, t_{2}}\right) \cup\left(\bar{\Omega} \times\left\{t_{1}\right\}\right) .
$$

We use the symbol $C$ to denote a constant and $C=C(\cdot)$ to describe the arguments of the constant. The constant may vary from line to line but the arguments are as 
in the statement of the theorem. For the sake of clarity, we enumerate different constants in some proofs.

We denote the standard mollification in the time direction of the function $f$ : $\mathbb{R}^{n} \times \mathbb{R} \mapsto \mathbb{R}$ as

$$
f_{h}(x, t)=\int_{\mathbb{R}} f(x, s) \zeta(h, t-s) d s,
$$

where $\zeta(h, s)$ is a standard mollifier, whose support is contained in $(-h, h), h>0$. If we have

$$
\lim _{h \downarrow 0} \int_{\Omega}\left(f_{h}(x, t)-f(x, t)\right)^{2} d x=0
$$

for some $t \in \mathbb{R}$ and $\Omega$ open in $\mathbb{R}^{n}$, we call $t$ as $\Omega$-Lebesgue instant of $u$, or simply Lebesgue instant.

We denote by $f_{+}$and $f_{-}$the non-negative and non-positive part of $f$, respectively. We use the abbreviation

$$
f_{\Omega} f d v=\frac{1}{v(\Omega)} \int_{\Omega} f d v
$$

for the averaged integral with respect to the measure $v$.

ACKNOWLEDGEMENTs. The author would like to thank Professor Juha Kinnunen for his many valuable comments and Juhana Siljander for his careful reading of the earlier version of the manuscript.

\section{Weak supersolutions}

We are now going to state our assumptions on $\mathcal{A}$ and define weak super- and subsolutions. Let $\Omega_{T}$ be a domain in $\mathbb{R}^{n} \times \mathbb{R}$. We assume that $\mathcal{A}: \Omega_{T} \times \mathbb{R} \times \mathbb{R}^{n} \mapsto \mathbb{R}^{n}$ is a Caratheodory function, that is, $(x, t) \mapsto \mathcal{A}(x, t, u, F)$ is measurable for every $(u, F)$ in $\mathbb{R} \times \mathbb{R}^{n}$ and $(u, F) \mapsto \mathcal{A}(x, t, u, F)$ is continuous for almost every $(x, t) \in \Omega_{T}$. We assume that the growth conditions

$$
\mathcal{A}(x, t, u, F) \cdot F \geq \mathcal{A}_{0}|F|^{p}, \quad \text { and } \quad|\mathcal{A}(x, t, u, F)| \leq \mathcal{A}_{1}|F|^{p-1},
$$

$p>2$, hold for every $(u, F) \in \mathbb{R} \times \mathbb{R}^{n}$ and for almost every $(x, t) \in \Omega_{T}$. Note that in our case the assumptions on the growth conditions are slightly less general than in [9], but this keeps the presentation more transparent. Positive constants $\mathcal{A}_{0}$, and $\mathcal{A}_{1}$ are called structural, or growth constants of $\mathcal{A}$. If $\mathcal{A}$ and $\widetilde{\mathcal{A}}$ are both as above with the same growth constants, we say that the corresponding equations are structurally similar.

We assume that the supersolutions belong to the parabolic Sobolev space. Suppose that $\Omega$ is a domain in $\mathbb{R}^{n}$. The Sobolev space $W^{1, p}(\Omega)$ is defined to be the 
space of real-valued functions $f$ such that $f \in L^{p}(\Omega)$ and the distributional first partial derivatives $\partial f / \partial x_{i}, i=1,2, \ldots, n$, exist in $\Omega$ and belong to $L^{p}(\Omega)$. We equip the Sobolev space with the norm

$$
\|f\|_{1, p, \Omega}=\left(\int_{\Omega}|f|^{p} d x\right)^{1 / p}+\left(\int_{\Omega}|\nabla f|^{p} d x\right)^{1 / p} .
$$

The Sobolev space with zero boundary values $W_{0}^{1, p}(\Omega)$ is the closure of $C_{0}^{\infty}(\Omega)$ with respect to the Sobolev norm. We denote by $L^{p}\left(t_{1}, t_{2} ; W^{1, p}(\Omega)\right), t_{1}<t_{2}$, the parabolic Sobolev space, which contains functions such that for almost every $t$, $t_{1}<t<t_{2}$, the function $x \mapsto u(x, t)$ belongs to $W^{1, p}(\Omega)$ and

$$
\|u\|_{L^{p}\left(t_{1}, t_{2} ; W^{1, p}(\Omega)\right)}=\left(\int_{t_{1}}^{t_{2}} \int_{\Omega}\left(|u(x, t)|^{p}+|\nabla u(x, t)|^{p}\right) d x d t\right)^{1 / p}<\infty .
$$

The definition of the space $L^{p}\left(t_{1}, t_{2} ; W_{0}^{1, p}(\Omega)\right)$ is analogous.

Definition 2.1. Let $\Xi$ be an open set in $\mathbb{R}^{n} \times \mathbb{R}$. We say that a function $u$ is a weak solution to (1.1) in $\Xi$, if for all open $\Omega_{t_{1}, t_{2}} \Subset \Xi$, we have $u \in L^{p}\left(t_{1}, t_{2} ; W^{1, p}(\Omega)\right.$ ) and

$$
-\int_{t_{1}}^{t_{2}} \int_{\Omega} u \frac{\partial \eta}{\partial t} d x d t+\int_{t_{1}}^{t_{2}} \int_{\Omega} \mathcal{A}(x, t, u, \nabla u) \cdot \nabla \eta d x d t=0
$$

for every test function $\eta \in C_{0}^{\infty}\left(\Omega_{t_{1}, t_{2}}\right)$.

A function $u$ is a weak supersolution (subsolution) to (1.1) in $\Xi$, if for all open $\Omega_{t_{1}, t_{2}} \Subset \Xi$, we have $u \in L^{p}\left(t_{1}, t_{2} ; W^{1, p}(\Omega)\right)$, and the left hand side of (2.2) is non-negative (non-positive) for all non-negative test functions $\eta \in C_{0}^{\infty}\left(\Omega_{t_{1}, t_{2}}\right)$.

If $u$ is a sub- or supersolution in an open set $\Xi$ which compactly contains $\Omega \times\left(t_{0}, t_{0}+T_{0}\right)$, then almost every $t_{0}<t<t_{0}+T_{0}$ is an $\Omega$-Lebesgue instant of $u$. This is because $u$ belongs to $L^{2}\left(t_{0}, t_{0}+T_{0} ; L^{2}(\Omega)\right)$.

Weak sub- and supersolutions admit a scaling property. Let $u$ be a weak supersolution (subsolution) to (1.1) in

$$
B\left(x_{0}, R_{0}\right) \times\left(t_{0}, t_{0}+T_{0}\right),
$$

where $x_{0} \in \mathbb{R}^{n}, t_{0} \in \mathbb{R}$ and $T_{0}, R_{0}>0$. Then it is an easy calculation to show that the scaled function

$$
v(\xi, \tau)=\frac{1}{\gamma} u\left(x_{1}+R \xi, t_{1}+T \tau\right), \quad \gamma=\left(\frac{R^{p}}{T}\right)^{1 /(p-2)},
$$

is a weak supersolution (subsolution) in

$$
B\left(\frac{x_{0}-x_{1}}{R}, \frac{R_{0}}{R}\right) \times\left(\frac{t_{0}-t_{1}}{T}, \frac{t_{0}-t_{1}}{T}+\frac{T_{0}}{T}\right)
$$


for every $R>0, T>0, x_{1} \in \mathbb{R}^{n}$ and $t_{1} \in \mathbb{R}$, to the structurally similar equation with

$$
\widetilde{\mathcal{A}}(\xi, \tau, v, \nabla v)=\left(\frac{R}{\gamma}\right)^{p-1} \mathcal{A}\left(x_{1}+R \xi, t_{1}+T \tau, \gamma v, \frac{\gamma}{R} \nabla v\right) .
$$

\subsection{Caccioppoli estimate}

In this section we extract all the needed information from the fact that the function $u$ is a weak supersolution. The obtained result is a consequence of a substitution of a suitable test function in (2.2). More precisely, the choice depends on $u$ itself. It is clear that the test function chosen this way is not necessarily smooth, or not even a Sobolev function. The time derivative of $u$ is, in general, merely a generalized function. Nevertheless, we may regularize the weak supersolution by using either Friedrich's mollifiers, Steklov averages or some other suitable method. Together with the truncation and approximation argument this justifies the choice of such a test function.

The following Caccioppoli estimate is one of the key estimates of this work. Also the byproduct (2.3) will be used in future.

Lemma 2.2. Let $\varepsilon \in \mathbb{R} \backslash\{-1,0\}$ and $\delta>0$. Suppose that $u \geq \delta$ is a weak subsolution (if $\varepsilon>0$ ) or a weak supersolution (if $\varepsilon<0$ ) in $\Omega_{\tau_{1}, \tau_{2}}$. Then we have

$$
\begin{aligned}
\int_{\tau_{1}}^{\tau_{2}} \int_{\Omega}|\nabla u|^{p} u^{-1+\varepsilon} \varphi^{p} d x d t & +\frac{p}{\mathcal{A}_{0}|\varepsilon(1+\varepsilon)|} \underset{\tau_{1}<t<\tau_{2}}{\operatorname{ess} \sup } \int_{\Omega} u^{1+\varepsilon} \varphi^{p} d x \\
& \leq\left(\frac{\mathcal{A}_{1} p}{\mathcal{A}_{0} \min \{1,|\varepsilon|\}}\right)^{p} \int_{\tau_{1}}^{\tau_{2}} \int_{\Omega} u^{p-1+\varepsilon}|\nabla \varphi|^{p} d x d t \\
& +\frac{p}{\mathcal{A}_{0}} \int_{\tau_{1}}^{\tau_{2}} \int_{\Omega} u^{1+\varepsilon}\left(\frac{1}{\min \{1, \varepsilon\}(1+\varepsilon)} \frac{\partial \varphi^{p}}{\partial t}\right)_{+} d x d t
\end{aligned}
$$

for every non-negative $\varphi \in C_{0}^{\infty}\left(\Omega_{\tau_{1}, \tau_{2}}\right)$.

Proof. We first fix $\varphi \in C_{0}^{\infty}\left(\Omega_{\tau_{1}, \tau_{2}}\right)$ and choose $h$ to be small enough so that the support of $\varphi_{h}$ does not intersect the Euclidean boundary of $\Omega_{\tau_{1}, \tau_{2}}$. Here the subscript $h$ refers to the standard mollification, see Section 1.1. We formally choose the test function $\eta=\left(\psi \theta_{j}\right)_{h}$, where

$$
\psi=\min \left\{u_{h}^{-1+\varepsilon}, k^{|-1+\varepsilon|}\right\} u_{h} \varphi^{p}, \quad k^{|-1+\varepsilon|}>\delta^{-1+\varepsilon},
$$

and $\theta_{j} \in C_{0}^{\infty}\left(\tau_{1}, \tau_{2}\right), j=1,2, \ldots$, converges to the characteristic function of the interval $\left(t_{1}, t_{2}\right), \tau_{1}<t_{1}<t_{2}<\tau_{2}$, in $L^{p}$ as $j \rightarrow \infty$. The test function $\eta$ is admissible due to the approximation in $L^{p}\left(\tau_{1}, \tau_{2} ; W_{0}^{1, p}(\Omega)\right)$ by compactly supported smooth functions, and, the fact that we may temporarily redefine $u_{h}$ to be zero outside the support of $\varphi$. Note that if $\varepsilon \leq 1$, then $\psi=u_{h}^{\varepsilon} \varphi^{p}$. We substitute the test 
function $\eta$ into (2.2), change variables, apply Fubini's theorem, integrate by parts and let $j \rightarrow \infty$. We obtain the following regularized integral inequality

$$
\int_{t_{1}}^{t_{2}} \int_{\Omega} \frac{\partial u_{h}}{\partial t} \psi d x d t+\int_{t_{1}}^{t_{2}} \int_{\Omega}(\mathcal{A}(x, t, u, \nabla u))_{h} \cdot \nabla \psi d x d t \geq(\leq) 0
$$

for every $\tau_{1}<t_{1}<t_{2}<\tau_{2}$. It follows by the properties of standard mollifiers that

$$
(\mathcal{A}(x, t, u, \nabla u))_{h} \cdot \nabla \psi \rightarrow \mathcal{A}(x, t, u, \nabla u) \cdot \nabla\left(\min \left\{u^{-1+\varepsilon}, k^{|-1+\varepsilon|}\right\} u \varphi^{p}\right)
$$

in $L^{1}\left(\Omega_{t_{1}, t_{2}}\right)$ as $h \rightarrow 0$. When $\varepsilon \leq 1$, or $\varepsilon>1$ and $u<k$, the growth conditions (2.1) imply that for almost every $(x, t)$ in the support of $\varphi$, we have

$$
u^{\varepsilon-1} \varphi^{p} \mathcal{A}(x, t, u, \nabla u) \cdot \nabla u \geq \mathcal{A}_{0}|\nabla u|^{p} u^{\varepsilon-1} \varphi^{p}
$$

and

$$
\begin{aligned}
& \frac{p}{\varepsilon} u^{\varepsilon} \varphi^{p-1} \mathcal{A}(x, t, u, \nabla u) \cdot \nabla \varphi \geq-\frac{\mathcal{A}_{1} p}{|\varepsilon|}|\nabla u|^{p-1} \varphi^{p-1}|\nabla \varphi| u^{\varepsilon} \\
& =-\mathcal{A}_{0}\left(|\nabla u| u^{(-1+\varepsilon) / p} \varphi\right)^{p-1}\left(\frac{\mathcal{A}_{1} p}{\mathcal{A}_{0}|\varepsilon|} u^{(p-1+\varepsilon) / p}|\nabla \varphi|\right) \\
& \geq-\frac{(p-1)}{p} \mathcal{A}_{0}|\nabla u|^{p} u^{-1+\varepsilon} \varphi^{p}-\frac{\mathcal{A}_{0}}{p}\left(\frac{\mathcal{A}_{1} p}{\mathcal{A}_{0}|\varepsilon|}\right)^{p} u^{p-1+\varepsilon}|\nabla \varphi|^{p} .
\end{aligned}
$$

Here we have also applied Young's inequality. Therefore,

$$
\frac{p}{\mathcal{A}_{0} \varepsilon} \mathcal{A}(x, t, u, \nabla u) \cdot \nabla\left(u^{\varepsilon} \varphi^{p}\right) \geq|\nabla u|^{p} u^{-1+\varepsilon} \varphi^{p}-\left(\frac{\mathcal{A}_{1} p}{\mathcal{A}_{0}|\varepsilon|}\right)^{p} u^{p-1+\varepsilon}|\nabla \varphi|^{p}
$$

for almost every $(x, t)$ in the support of $\varphi$. Similarly, when $\varepsilon>1$ and $u \geq k$, we have

$$
\frac{p}{\mathcal{A}_{0}} \mathcal{A}(x, t, u, \nabla u) \cdot \nabla\left(k^{-1+\varepsilon} u \varphi^{p}\right) \geq|\nabla u|^{p} k^{-1+\varepsilon} \varphi^{p}-\left(\frac{\mathcal{A}_{1} p}{\mathcal{A}_{0}}\right)^{p} u^{p} k^{-1+\varepsilon}|\nabla \varphi|^{p}
$$

for almost every $(x, t)$ in the support of $\varphi$.

Furthermore, we set

$$
g(s)=\int_{\delta}^{s} \min \left\{r^{-1+\varepsilon}, k^{|-1+\varepsilon|}\right\} r d r .
$$

We integrate by parts and obtain

$$
\begin{aligned}
\int_{t_{1}}^{t_{2}} \int_{\Omega} \frac{\partial u_{h}}{\partial t} \psi d x d t & =\int_{t_{1}}^{t_{2}} \int_{\Omega} \frac{\partial g\left(u_{h}\right)}{\partial t} \varphi^{p} d x d t \\
& =-\int_{t_{1}}^{t_{2}} \int_{\Omega} g\left(u_{h}\right) \frac{\partial \varphi^{p}}{\partial t} d x d t+\left.\int_{\Omega} g\left(u_{h}(x, t)\right) \varphi^{p}(x, t) d x\right|_{t=t_{1}} ^{t_{2}}
\end{aligned}
$$


for every $\tau_{1}<t_{1}<t_{2}<\tau_{2}$. Let then $t$ be a Lebesgue instant. We have

$$
\begin{aligned}
& \int_{\Omega} g\left(u_{h}(x, t)\right) \varphi^{p}(x, t) d x \\
& =\frac{\delta^{1+\varepsilon}}{1+\varepsilon} \int_{\Omega} \varphi^{p}(x, t) d x+\int_{\left\{x \in \Omega: u_{h}^{\operatorname{sgn}(-1+\varepsilon)}(x, t) \leq k\right\}} \frac{u_{h}^{1+\varepsilon}(x, t)}{1+\varepsilon} \varphi^{p}(x, t) d x \\
& \quad+\int_{\left\{x \in \Omega: u_{h}^{\operatorname{sgn}(-1+\varepsilon)}(x, t)>k\right\}} k^{-1+\varepsilon}\left(\frac{k^{2}}{1+\varepsilon}+\frac{u_{h}^{2}(x, t)-k^{2}}{2}\right) \varphi^{p}(x, t) d x .
\end{aligned}
$$

Since $t$ is a Lebesgue instant, $u_{h}(x, t) \rightarrow u(x, t)$ for almost every $x$ in the support of $\varphi(\cdot, t)$ as $h \rightarrow 0$. On the one hand, we obtain by the dominated convergence theorem that

$$
\begin{aligned}
& \int_{\left\{x \in \Omega: u_{h}^{\operatorname{sgn}(-1+\varepsilon)}(x, t) \leq k\right\}} \frac{u_{h}^{1+\varepsilon}(x, t)}{1+\varepsilon} \varphi^{p}(x, t) d x \\
& \rightarrow \int_{\left\{x \in \Omega: u^{\operatorname{sgn}(-1+\varepsilon)}(x, t) \leq k\right\}} \frac{u^{1+\varepsilon}(x, t)}{1+\varepsilon} \varphi^{p}(x, t) d x
\end{aligned}
$$

as $h \rightarrow 0$. On the other hand,

$$
\begin{aligned}
& \int_{\left\{x \in \Omega: u_{h}^{\operatorname{sgn}(-1+\varepsilon)}(x, t)>k\right\}}\left(u_{h}-u\right)^{2}(x, t) \varphi^{p}(x, t) d x \\
& \leq\|\varphi\|_{\infty}^{p} \int_{\Omega}\left(u_{h}-u\right)^{2}(x, t) d x \rightarrow 0
\end{aligned}
$$

as $h \rightarrow 0$ by the definition of Lebesgue instant. We conclude that

$$
\begin{aligned}
& \int_{t_{1}}^{t_{2}} \int_{\Omega} \frac{\partial u_{h}}{\partial t} \psi d x d t \\
& \rightarrow-\int_{t_{1}}^{t_{2}} \int_{\Omega} g(u) \frac{\partial \varphi^{p}}{\partial t} d x d t+\left.\int_{\Omega} g(u(x, t)) \varphi^{p}(x, t) d x\right|_{t=t_{1}} ^{t_{2}}
\end{aligned}
$$

as $h \rightarrow 0$ for all Lebesgue instants $\tau_{1}<t_{1}<t_{2}<\tau_{2}$.

We combine the obtained estimates and divide the result by $\mathcal{A}_{0} \varepsilon / p$. As $k \rightarrow$ $\infty$, the monotone convergence theorem implies

$$
\begin{aligned}
0 \geq & \int_{t_{1}}^{t_{2}} \int_{\Omega}|\nabla u|^{p} u^{-1+\varepsilon} \varphi^{p} d x d t \\
& -\left(\frac{\mathcal{A}_{1} p}{\mathcal{A}_{0} \min \{1,|\varepsilon|\}}\right)^{p} \int_{t_{1}}^{t_{2}} \int_{\Omega} u^{p-1+\varepsilon}|\nabla \varphi|^{p} d x d t \\
& -\frac{p}{\mathcal{A}_{0}} \int_{t_{1}}^{t_{2}} \int_{\Omega} u^{1+\varepsilon}\left(\frac{1}{\min \{1, \varepsilon\}(1+\varepsilon)} \frac{\partial \varphi^{p}}{\partial t}\right)_{+} d x d t \\
& +\left.\frac{p}{\mathcal{A}_{0} \min \{1, \varepsilon\}(1+\varepsilon)} \int_{\Omega} u^{1+\varepsilon}(x, t) \varphi^{p}(x, t) d x\right|_{t=t_{1}} ^{t_{2}}
\end{aligned}
$$


for all Lebesgue instants $\tau_{1}<t_{1}<t_{2}<\tau_{2}$.

Let then $\rho>0$. We may choose $\tau_{1}<t_{i}<\tau_{2}, i=1,2$, such that

$$
\int_{\Omega} u^{1+\varepsilon}\left(x, t_{i}\right) \varphi^{p}\left(x, t_{i}\right) d x \geq \underset{\tau_{1}<t<\tau_{2}}{\operatorname{ess} \sup } \int_{\Omega} u^{1+\varepsilon} \varphi^{p} d x-\rho,
$$

$i=1$, 2. If $\varepsilon(1+\varepsilon)>0$, we choose $t_{2}$ and let $t_{1} \rightarrow \tau_{1}$, and, if $\varepsilon(1+\varepsilon)<0$, we choose $t_{1}$ and let $t_{2} \rightarrow \tau_{2}$. This concludes the proof, since $\rho$ is arbitrary.

Remark 2.3. If $u$ is a weak supersolution, then $(u-k)_{-}=\max \{k-u, 0\}, k \in \mathbb{R}$, is a non-negative weak subsolution to an equation which is structurally similar to the equation of $u$. Thus we may first apply the previous result for the weak subsolution $v=(u-k)_{-}+\delta, \delta>0$, and then use the dominated convergence theorem and let $\delta \rightarrow 0$. This implies that $u$ may be replaced by $(u-k)_{-}$in the statement of the lemma and in (2.3). Indeed, by choosing $\varepsilon=1$, we obtain the classical energy estimate for weak supersolutions.

Remark 2.4. If $u$ is a non-negative weak supersolution in an open set which compactly contains $\Omega_{t_{1}, t_{2}}$, where $t_{1}$ and $t_{2}$ are Lebesgue instants, then there is $\tau>0$ such that $u$ is a weak supersolution also in $\Omega \times\left(t_{1}-\tau, t_{2}+\tau\right)$. We choose the test function $\eta=\left(\varphi \theta_{j}\right)_{h}, h<\tau / 2$, where $\varphi \in C_{0}^{\infty}(\Omega)$ and $\theta_{j} \in C_{0}^{\infty}\left(t_{1}-\tau+h, t_{2}+\right.$ $\tau-h), j=1,2, \ldots$, converges to the characteristic function of the interval $\left(t_{1}, t_{2}\right)$ in $L^{p}$ as $j \rightarrow \infty$. We may then proceed as in the proof of Lemma 2.2 and obtain

$$
\begin{aligned}
\int_{\Omega} u\left(x, t_{2}\right) \varphi(x) d x \geq & \int_{\Omega} u\left(x, t_{1}\right) \varphi(x) d x \\
& +\int_{t_{1}}^{t_{2}} \int_{\Omega} \mathcal{A}(x, t, u, \nabla u) \cdot \nabla \varphi d x d t .
\end{aligned}
$$

\section{Expansion of positivity}

A fundamental property of a supersolution to a diffusion equation is that the positivity expands as the time evolve. The following proposition describes the phenomenon.

Proposition 3.1 (Expansion of positivity). Let u be a non-negative weak supersolution in an open set which compactly contains $B\left(x_{0}, 4 R_{0}\right) \times\left(t_{0}, t_{0}+T_{0}\right)$. Suppose that $t_{0}$ is a Lebesgue instant and

$$
\left|\left\{x \in B\left(x_{0}, R\right): u\left(x, t_{0}\right)>N\right\}\right| \geq \delta\left|B\left(x_{0}, R\right)\right|
$$

for some $0<R<R_{0}, N>0$ and $0<\delta<1$. Then there are positive constants $\bar{T}=\bar{T}\left(n, p, \mathcal{A}_{0}, \mathcal{A}_{1}, \delta\right)$ and $\theta=\theta\left(n, p, \mathcal{A}_{0}, \mathcal{A}_{1}, \delta\right)$ such that if

$$
T=\bar{T}\left(N\left(R / R_{0}\right)^{\theta}\right)^{2-p} R_{0}^{p}
$$


does not exceed $T_{0}$, then

$$
\underset{Q}{\operatorname{ess} \inf } u \geq N\left(R / R_{0}\right)^{\theta},
$$

where $Q=B\left(x_{0}, 2 R_{0}\right) \times\left(t_{0}+T / 2, t_{0}+T\right)$.

The idea of the proof is the following. We first carry the assumed positivity further in time. We show that for some Lebesgue instant, the spatial level set of the supersolution has positive Lebesgue measure and a finite capacity type constraint with respect to a larger level set. Next, positive values of the supersolution may decay in time. We cancel the decay by simply multiplying the supersolution by the inverse of the decay factor. It follows from the change of the time variable that the new function is a supersolution to an equation which is structurally similar to the equation of the original supersolution. It is then a fairly standard argument to show that the positivity expands in time. The main real analytical tools for the proof can be found from [8], see also [12] or [28]. Note that the stability of constants as $p \rightarrow 2$ is inbuilt to our approach. In particular, there is no need to separate the cases $p$ close to 2 and $p$ away from 2 .

We prove Proposition 3.1 by using a scaling argument. This leads us to study a supersolution $u$ in an open set which compactly contains $B(0,4) \times\left(0, T_{\text {pos }}\right)$ such that 0 is a Lebesgue instant and

$$
|\{x \in B(0,1): u(x, 0)>N\}| \geq \delta|B(0,1)|
$$

for some $N>0$ and $0<\delta<1$.

The first lemma yields that the assumed positivity at the instant 0 can be transferred to later times.

Lemma 3.2. Let $k>0$ and $0<\gamma<1$. Then there is a constant $C=C\left(n, p, \mathcal{A}_{0}, \mathcal{A}_{1}\right)$ such that if $u$ is a non-negative weak supersolution in an open set which compactly contains $B\left(x_{0}, 2 R\right) \times\left(t_{0}, t_{0}+k^{2-p} \gamma^{p+1} R^{p} / C\right)$, where $t_{0}$ is a Lebesgue instant and

$$
\left|\left\{x \in B\left(x_{0}, R\right): u\left(x, t_{0}\right)>k\right\}\right| \geq \gamma\left|B\left(x_{0}, R\right)\right|,
$$

then

$$
\left|\left\{x \in B\left(x_{0}, R\right): u(x, t)>\frac{\gamma}{8} k\right\}\right| \geq \frac{\gamma}{8}\left|B\left(x_{0}, R\right)\right|
$$

holds for all Lebesgue instants $t_{0}<t<t_{0}+k^{2-p} \gamma^{p+1} R^{p} / C$.

Proof. Let $\varepsilon=\gamma /(5 n)$ and $T=k^{2-p} \gamma^{p+1} R^{p} / C$. Let $\varphi$ be a cut-off function in $C_{0}^{\infty}\left(B\left(x_{0},(1+\varepsilon) R\right)\right)$ such that $\varphi=1$ in $B\left(x_{0}, R\right), 0 \leq \varphi \leq 1$ and $|\nabla \varphi| \leq$ $C_{1} /(\varepsilon R)$. By Remark 2.3 and the facts that $u$ is a supersolution in an open set which compactly contains $B\left(x_{0}, 2 R\right) \times\left(t_{0}, t_{0}+T\right)$ and that $t_{0}$ is a Lebesgue instant, we may insert $\varphi$ and $(u-k)_{-}$into (2.3). We obtain

$$
\begin{aligned}
\int_{B\left(x_{0}, R\right)}(u(x, \tau)-k)_{-}^{2} d x \leq & \int_{B\left(x_{0},(1+\varepsilon) R\right)}\left(u\left(x, t_{0}\right)-k\right)_{-}^{2} d x \\
& +C_{2} \int_{0}^{T} \int_{B\left(x_{0},(1+\varepsilon) R\right)}(u-k)_{-}^{p}|\nabla \varphi|^{p} d x d t
\end{aligned}
$$


for all Lebesgue instants $t_{0}<\tau<t_{0}+T$. Using the assumption, we estimate the first term on the right hand side as

$$
\begin{aligned}
& \int_{B\left(x_{0},(1+\varepsilon) R\right)}\left(u\left(x, t_{0}\right)-k\right)_{-}^{2} \varphi^{p} d x \\
& \leq k^{2}\left|B\left(x_{0},(1+\varepsilon) R\right) \backslash B\left(x_{0}, R\right)\right|+\int_{B\left(x_{0}, R\right)}\left(u\left(x, t_{0}\right)-k\right)_{-}^{2} \varphi^{p} d x \\
& \leq\left(\left((1+\varepsilon)^{n}-1\right) k^{2}+(1-\gamma) k^{2}\right)\left|B\left(x_{0}, R\right)\right| \\
& \leq(1-3 \gamma / 4) k^{2}\left|B\left(x_{0}, R\right)\right|,
\end{aligned}
$$

because $(1+\varepsilon)^{n}-1 \leq n \varepsilon /(1-n \varepsilon) \leq \gamma / 4$. The second term we estimate as

$$
C_{2} \int_{0}^{T} \int_{B\left(x_{0},(1+\varepsilon) R\right)}(u-k)_{-}^{p}|\nabla \varphi|^{p} d x d t \leq \frac{C_{3} k^{p} T}{(\varepsilon R)^{p}}\left|B\left(x_{0}, R\right)\right| .
$$

For the left hand side we have

$$
\int_{B\left(x_{0}, R\right)}(u(x, \tau)-k)_{-}^{2} d x \geq(1-\gamma / 8)^{2} k^{2}\left|\left\{x \in B\left(x_{0}, R\right): u(x, \tau) \leq \frac{\gamma}{8} k\right\}\right|
$$

for every Lebesgue instant $t_{0}<\tau<t_{0}+T$. We then choose the constant $C$ in the statement to be large enough so that

$$
\frac{C_{3} k^{p} T}{(\varepsilon R)^{p}}=\frac{C_{3} k^{p} k^{2-p} R^{p} \gamma^{p+1} / C}{\gamma^{p} /(5 n)^{p} R^{p}}=\frac{(5 n)^{p} C_{3} \gamma k^{2}}{C} \leq \frac{\gamma}{4} k^{2} .
$$

We conclude that

$$
\left|\left\{x \in B\left(x_{0}, R\right): u(x, \tau) \leq \frac{\gamma}{8} k\right\}\right| \leq \frac{1-\gamma / 2}{1-\gamma / 4}\left|B\left(x_{0}, R\right)\right|
$$

for all Lebesgue instants $t_{0}<\tau<t_{0}+T$. This proves the result.

Next, we show that from the obtained time range, we find a Lebesgue instant such that we have the control of the capacity type of constraint between two level sets.

Lemma 3.3. Let $u$ be a non-negative weak supersolution in an open set which compactly contains $B(0,4) \times\left(0, T_{\mathrm{pos}}\right)$ and suppose that 0 is a Lebesgue instant. There are constants $C_{i}=C_{i}\left(n, p, \mathcal{A}_{0}, \mathcal{A}_{1}, \delta\right), i=1,2$, such that if $T_{\mathrm{pos}}>1 /\left(C_{1} N^{p-2}\right)$ and

$$
|\{x \in B(0,1): u(x, 0)>N\}| \geq \delta|B(0,1)|, \quad N>0, \quad 0<\delta<1,
$$

then there are a Lebesgue instant $0<t^{*}<1 /\left(C_{1} N^{p-2}\right)$ and a Sobolev function

$$
\psi \in W_{0}^{1, p}(B(0,2)), \quad 0 \leq \psi \leq 1,
$$


such that the positivity is carried up to $t^{*}$, i.e.

$$
\left|\left\{x \in B(0,1): u\left(x, t^{*}\right)>\frac{\delta}{8} N\right\}\right| \geq \frac{\delta}{8}|B(0,1)|,
$$

and the function $\psi$ measures capacity type of constraint between two level sets of $u\left(\cdot, t^{*}\right)$, i.e.

$$
\begin{aligned}
& \psi=1 \text { almost everywhere in } \quad\left\{x \in B(0,1): u\left(x, t^{*}\right)>\frac{\delta}{8} N\right\}, \\
& \psi=0 \text { almost everywhere in } \quad\left\{x \in B(0,2): u\left(x, t^{*}\right) \leq \frac{\delta}{16} N\right\}
\end{aligned}
$$

and

$$
\int_{B(0,2)}|\nabla \psi|^{p} d x \leq C_{2}
$$

Proof. Let $k=N \delta / 8$. We choose $T=1 /\left(2 C_{1} N^{p-2}\right)$ and assume that $T<T_{\text {pos }} / 2$. Let then $\varphi \in C_{0}^{\infty}(B(0,2) \times(0,2 T)), 0 \leq \varphi \leq 1$, be a cut-off function such that $\varphi=1$ in $B(0,1) \times(T, 2 T)$ and $|\nabla \varphi|,(\partial \varphi / \partial t)_{+} \leq C / T$. By Remark 2.3 we may apply Lemma 2.2 for $v=(2 k-u)_{+}$and $\varepsilon=1$, and obtain that there is a constant $C=C\left(n, p, \mathcal{A}_{0}, \mathcal{A}_{1}\right)$ such that

$$
\int_{T / 2}^{T} \int_{B(0,2)}|\nabla(v \varphi)|^{p} d x d t \leq C\left(k^{p} T+k^{2}\right)
$$

Furthermore, the function

$$
w=\frac{1}{k}(k-v)_{+}=\frac{1}{k}\left(k-(2 k-u)_{+}\right)_{+}
$$

vanishes in $\{u \leq k\}$ and $w=1$ in $\{u \geq 2 k\}$. Moreover, we have

$$
\int_{T / 2}^{T} \int_{B(0,2)}|\nabla(w \varphi)|^{p} d x d t \leq \frac{1}{k^{p}} \int_{T / 2}^{T} \int_{B(0,2)}|\nabla(v \varphi)|^{p} d x d t \leq C\left(T+k^{2-p}\right) .
$$

Therefore, there exists a Lebesgue instant $T / 2<t^{*}<T$ such that

$$
\int_{B(0,2)}\left|\nabla(w \varphi)\left(x, t^{*}\right)\right|^{p} d x \leq C\left(1+\frac{1}{T k^{p-2}}\right) \leq C .
$$

Thus we may choose $\psi=w\left(\cdot, t^{*}\right) \varphi\left(\cdot, t^{*}\right)$.

The first part concerning the measure estimate follows immediately from Lemma 3.2, if $C_{1}$ is chosen to be large enough. 
The next lemma is a straightforward consequence of a choice of a proper test function. Note that when $p=2$, the choice is $u^{-1}$ and it leads to the logarithmic estimate, which is the cornerstone of the proof of the Main Lemma in [24].

Lemma 3.4. Let $u$ and $t^{*}$ be as in Lemma 3.3. Then there exist constants $\kappa=$ $\kappa\left(n, p, \mathcal{A}_{0}, \mathcal{A}_{1}, \delta\right)$ and $v=v(n, \delta)$ such that for

$$
g(t)=\frac{\kappa}{N}\left(1+\kappa(p-2) N^{p-2} t\right)^{1 /(p-2)},
$$

we have

$$
|\{x \in B(0,3): u(x, t) g(t)>1\}| \geq v|B(0,3)|
$$

for all Lebesgue instants $t^{*}<t<T_{\text {pos }}$.

Proof. First, we define the supersolution $v=u+\rho, \rho>0$. Let $\psi \in W_{0}^{1, p}(B(0,2))$ be as in Lemma 3.3. We substitute $\varepsilon=1-p$ and $\varphi=\psi$ into (2.3) and obtain

$$
\begin{aligned}
\frac{1}{p-2} \int_{B(0,2)} v^{2-p}(x, t) \psi^{p} d x \leq & \frac{1}{p-2} \int_{B(0,2)} v^{2-p}\left(x, t^{*}\right) \psi^{p} d x \\
& +C t \int_{B(0,2)}|\nabla \psi|^{p} d x
\end{aligned}
$$

for all Lebesgue instants $t^{*}<t<T_{\text {pos. }}$. The substitution is possible due to the approximation of $\psi$ in $C_{0}^{\infty}(B(0,2))$ and the fact that $t^{*}$ is a Lebesgue instant. Since $v\left(\cdot, t^{*}\right) \geq N \delta / 16+\rho$ almost everywhere in the support of $\psi$, we get

$$
\int_{B(0,2)} v^{2-p}\left(x, t^{*}\right) \psi^{p}(x) d x \leq \int_{B(0,2)}(N \delta / 16)^{2-p} \psi^{p}(x) d x .
$$

By the monotone convergence theorem and the $L^{p}$-bound for $\nabla \psi$, we may send $\rho$ to zero, and conclude

$$
\int_{B(0,2)} \frac{u^{2-p}(x, t)-(N \delta / 16)^{2-p}}{p-2} \psi^{p}(x) d x \leq C t
$$

for all Lebesgue instants $t^{*}<t<T_{\text {pos }}$.

Next, we denote

$$
U=\left\{x \in B(0,1): u\left(x, t^{*}\right) \geq \frac{\delta}{8} N\right\} .
$$

In $U$ we have $\psi=1$ almost everywhere. This implies that for every $\gamma>0$ and for every Lebesgue instant $t^{*}<t<T_{\text {pos }}$, we have

$$
\begin{aligned}
& |\{x \in U: u(x, t) \leq \gamma\}| \frac{\gamma^{2-p}-(N \delta / 16)^{2-p}}{p-2} \\
& \leq \int_{B(0,2)} \frac{u^{2-p}(x, t)-(N \delta / 16)^{2-p}}{p-2} \psi^{p}(x) d x \leq C t .
\end{aligned}
$$


We then choose

$$
\gamma=\gamma(t)=\frac{N \delta}{16}\left(1+2 C(p-2)\left(\frac{N \delta}{16}\right)^{p-2} \frac{t}{|U|}\right)^{-1 /(p-2)}
$$

and obtain

$$
|\{x \in U: u(x, t) \leq \gamma(t)\}| \leq \frac{1}{2}|U| .
$$

This together with the measure estimate in Lemma 3.3 implies

$$
|\{x \in B(0,3): u(x, t)>\gamma(t)\}| \geq \frac{1}{2}|U| \geq \frac{\delta}{16}|B(0,1)| .
$$

The result now follows with constants $\kappa=C / \delta$ and $v=\delta / 3^{n+3}$. Note that they stay bounded as $p \rightarrow 2$.

A crucial step in the proof of Proposition 3.1 is that the function $g(t) u(x, t)$ is, after a proper change of the time variable, a supersolution.

Lemma 3.5. Suppose that $u$ is as in Lemma 3.3 and let

$$
\Lambda(t)=\frac{1}{\kappa(p-2)} \log \left(1+\kappa(p-2) N^{p-2} t\right)
$$

where $\kappa, N>0$. Then

$$
v(x, t)=\frac{\exp (\kappa t)}{N} u\left(x, \Lambda^{-1}(t)\right)
$$

is a weak supersolution in $B(0,4) \times\left(0, \Lambda\left(T_{\mathrm{pos}}\right)\right)$ to an equation, which is structurally similar to the equation of $u$.

Proof. Let $\varphi \in C_{0}^{\infty}\left(B(0,4) \times\left(0, \Lambda\left(T_{\text {pos }}\right)\right)\right.$ be a non-negative test function. We first define

$$
g(t)=\frac{1}{N}\left(1+\kappa(p-2) N^{p-2} t\right)^{1 /(p-2)}
$$

and set

$$
\eta(x, t)=g(t) \varphi(x, \Lambda(t)), \quad v(x, t)=g\left(\Lambda^{-1}(t)\right) u\left(x, \Lambda^{-1}(t)\right)
$$

for all $(x, t) \in B(0,4) \times\left(0, T_{\text {pos }}\right)$. Here

$$
\Lambda^{-1}(t)=\frac{\exp (\kappa(p-2) t)-1}{\kappa(p-2) N^{p-2}}
$$

We denote

$$
\tilde{\varphi}(x, t)=\varphi(x, \Lambda(t))=\eta(x, t) / g(t), \quad \widetilde{v}(x, t)=v(x, \Lambda(t))=g(t) u(x, t)
$$


for all $(x, t) \in B(0,4) \times\left(0, T_{\text {pos }}\right)$. The definitions imply that

$$
\nabla u=\frac{1}{g} \nabla \widetilde{v}, \quad u \frac{\partial \eta}{\partial t}=\frac{\kappa N^{p-2}}{1+\kappa(p-2) N^{p-2} t} \widetilde{v} \widetilde{\varphi}+\widetilde{v} \frac{\partial \widetilde{\varphi}}{\partial t} .
$$

Furthermore, we set

$$
\widetilde{\mathcal{A}}(x, t, \widetilde{v}, \nabla \widetilde{v})=g^{p-1} \mathcal{A}(x, t, \widetilde{v} / g, \nabla \widetilde{v} / g),
$$

and obtain

$$
\widetilde{\mathcal{A}}(x, t, \widetilde{v}, \nabla \widetilde{v}) \cdot \nabla \widetilde{v} \geq \mathcal{A}_{0}|\nabla \widetilde{v}|^{p}, \quad|\widetilde{\mathcal{A}}(x, t, \tilde{v}, \nabla \widetilde{v})| \leq \mathcal{A}_{1}|\nabla \widetilde{v}|^{p-1}
$$

and

$$
\mathcal{A}(x, t, u, \nabla u) \cdot \nabla \eta=\frac{N^{p-2}}{1+\kappa(p-2) N^{p-2} t} \widetilde{\mathcal{A}}(x, t, \tilde{v}, \nabla \widetilde{v}) \cdot \nabla \widetilde{\varphi} .
$$

According to the formula $t=\Lambda^{-1}(\tau)$, we have

$$
d \tau=\frac{N^{p-2}}{1+\kappa(p-2) N^{p-2} t} d t .
$$

We substitute the calculations into (2.2) and arrive at

$$
\begin{aligned}
0 \leq & \int_{0}^{\Lambda\left(T_{\mathrm{pos}}\right)} \int_{B(0,4)} \tilde{\mathcal{A}}\left(x, \Lambda^{-1}(\tau), v, \nabla v\right) \cdot \nabla \varphi d x d \tau \\
& -\int_{0}^{\Lambda\left(T_{\mathrm{pos}}\right)} \int_{B(0,4)} v \frac{\partial \varphi}{\partial \tau} d x d \tau-\kappa \int_{0}^{\Lambda\left(T_{\mathrm{pos}}\right)} \int_{B(0,4)} v \varphi d x d \tau .
\end{aligned}
$$

It follows by the non-negativity of $u$, and hence also $v$, that $v$ is a weak supersolution.

We have an immediate corollary of Lemma 3.4 and Lemma 3.5.

Corollary 3.6. Let $u$ be as in Lemma 3.3, $\kappa$ and $v$ as in Lemma 3.4 and $\Lambda$ as in (3.1). Then there is a constant $0<\tau^{*}<1$ such that the weak supersolution

$$
v(x, t)=\frac{\exp (\kappa t)}{N} u\left(x, \Lambda^{-1}(t)\right)
$$

in $B(0,4) \times\left(0, \Lambda\left(T_{\mathrm{pos}}\right)\right)$ satisfies

$$
|\{x \in B(0,3): v(x, t)>1\}| \geq v|B(0,3)|
$$

for almost every $\tau^{*}<t<\Lambda\left(T_{\mathrm{pos}}\right)$. 
Proof. We define $\tau^{*}=\Lambda\left(t^{*}\right)$, where $t^{*}$ is as in Lemma 3.3. The upper bound $1 /\left(C_{1} N^{p-2}\right)$ for $t^{*}$ implies $\tau^{*} \leq 1 / C_{1}<1$. The result now follows by Lemma 3.4 and Lemma 3.5.

We use the corollary above to show that for later times, the small values of $u$ lie in a set which has as small a measure as we please. This type of argument has been used in 'Second Alternative' in the proof of the Hölder continuity of weak solutions, see [8].

Lemma 3.7. Let $v$ be as in Corollary 3.6. Then, for every $0<v^{*}<1$, there exists a constant $M=M\left(n, p, \mathcal{A}_{0}, \mathcal{A}_{1}, v^{*}\right)$ such that if $T=2^{1+M(p-2)}<\Lambda\left(T_{\mathrm{pos}}\right) / 4$, where $\Lambda$ is as in (3.1), then

$$
\left|\left\{(x, t) \in B(0,3) \times(T, 4 T): v(x, t) \leq 2^{-M}\right\}\right| \leq v^{*}|B(0,3) \times(T, 4 T)| .
$$

Proof. We define $k_{j}=2^{-j}, j=0,1,2, \ldots, M$, and $T=2 k_{M}^{2-p}<\Lambda\left(T_{\mathrm{pos}}\right) / 4$, where $M$ is going to be fixed. Let $\varphi$ be a test function vanishing on the parabolic boundary of $B(0,4) \times\left(\tau^{*}, 4 T\right)$, where $\tau^{*}$ is as in Corollary 3.6, $\varphi=1$ in $B(0,3) \times$ $(T, 4 T)$ and $|\nabla \varphi|,(\partial \varphi / \partial t)_{+} \leq C$. Note that $T>2 \tau^{*}$. We estimate

$$
\begin{aligned}
& \int_{\tau^{*}}^{4 T} \int_{B(0,4)}\left(v-k_{j}\right)_{-}^{2}\left(\frac{\partial \varphi^{p}}{\partial t}\right)_{+} d x d t \\
& \leq \frac{C k_{j}^{2}}{T}|B(0,3) \times(T, 4 T)| \leq C k_{j}^{p}|B(0,3) \times(T, 4 T)|
\end{aligned}
$$

and

$$
\int_{\tau^{*}}^{4 T} \int_{B(0,4)}\left(v-k_{j}\right)_{-}^{p}|\nabla \varphi|^{p} d x d t \leq C k_{j}^{p}|B(0,3) \times(T, 4 T)| .
$$

It then follows from the energy estimate, see Remark 2.3, that

$$
\int_{T}^{4 T} \int_{B(0,3)}\left|\nabla\left(v-k_{j}\right)_{-}\right|^{p} d x d t \leq C k_{j}^{p}|B(0,3) \times(T, 4 T)| .
$$

A standard De Giorgi type of Sobolev's imbedding (see [8, Lemma 2.2, page 5]) together with Corollary 3.6 yields

$$
\begin{aligned}
& k_{j+1}\left|\left\{x \in B(0,3): v(x, t) \leq k_{j+1}\right\}\right| \\
& \leq C\left|\left\{x \in B(0,3): v(x, t) \geq k_{j}\right\}\right|^{-1} \int_{\left\{k_{j+1}<v(x, t)<k_{j}\right\}}|\nabla v|(x, t) d x \\
& \leq C \int_{\left\{k_{j+1}<v(x, t)<k_{j}\right\}}|\nabla v|(x, t) d x
\end{aligned}
$$


for almost every $\tau^{*}<t<\Lambda\left(T_{\text {pos }}\right)$ and $j=0,1, \ldots, M-1$. We integrate these in time from $T$ to $4 T$. The left hand side is bounded below by $k_{j+1}\left|\left\{v \leq k_{M}\right\}\right|$. Thus Hölder's inequality gives

$$
\begin{aligned}
& \left|\left\{(x, t) \in B(0,3) \times(T, 4 T): v(x, t) \leq k_{M}\right\}\right| \\
& \leq \frac{C}{k_{j+1}}\left(\int_{T}^{4 T} \int_{B(0,3)}\left|\nabla\left(v-k_{j}\right)_{-}\right|^{p} d x d t\right)^{1 / p} \\
& \quad \times\left(\int_{T}^{4 T} \int_{B(0,3)} \chi_{\left\{k_{j+1}<v<k_{j}\right\}} d x d t\right)^{(p-1) / p} \\
& \leq C(|B(0,3) \times(T, 4 T)|)^{1 / p}\left(\int_{T}^{4 T} \int_{B(0,3)} \chi_{\left\{k_{j+1}<v<k_{j}\right\}} d x d t\right)^{(p-1) / p} .
\end{aligned}
$$

We take the power $p /(p-1)$ on both sides and sum up from $j=0$ to $M-1$. Note that the sets $\left\{k_{j+1}<v<k_{j}\right\}$ are disjoint for different $j$ 's. This implies

$$
\left|\left\{(x, t) \in B(0,3) \times(T, 4 T): v(x, t) \leq k_{M}\right\}\right| \leq \frac{C}{M^{(p-1) / p}}|B(0,3) \times(T, 4 T)| .
$$

Therefore, by taking $M$ so large that

$$
M^{-(p-1) / p} \leq \frac{v^{*}}{C}
$$

we obtain the result.

We are ready to proceed to prove the preliminary version of Proposition 3.1. The proof is similar to the one of [8, Lemma 4.1, page 49].

Lemma 3.8. Let $u$ be as in Lemma 3.3. Then there exist constants $T^{*}$ and $\mu^{*}$ depending only on $n, p, \mathcal{A}_{0}, \mathcal{A}_{1}$, and $\delta$ such that if $N^{2-p} T^{*}<T_{\mathrm{pos}}$, then

$$
\underset{Q}{\operatorname{essinf}} u \geq N \mu^{*}
$$

where $Q=B(0,2) \times\left(N^{2-p} T^{*} / 2, N^{2-p} T^{*}\right)$. Moreover, constants $T^{*}$ and $\mu^{*}$ are stable as $p \rightarrow 2$.

Proof. Let $v$ be as in Corollary 3.6. By Lemma 3.7, we find for every $v^{*}$ a constant $M$ such that

$$
\left|\left\{(x, t) \in B(0,3) \times(T, 4 T): v(x, t) \leq 2^{-M}\right\}\right| \leq v^{*}|B(0,3) \times(T, 4 T)|,
$$

where $T=2^{1+M(p-2)}$. We define

$$
k_{j}=2^{-M-1}\left(1+2^{-j}\right), \quad r_{j}=2+2^{-j}, \quad T_{j}=2 T\left(1-2^{-j-1}\right)
$$


for $j=0,1,2, \ldots$ We then have

$$
k_{j}-k_{j+1}=2^{-M-j-2} \text { and } 2^{-M-1} \leq k_{j} \leq 2^{-M} .
$$

We also denote

$$
Q_{j}=B_{j} \times \Gamma_{j}=B\left(r_{j}, 0\right) \times\left(T_{j}, 4 T\right) .
$$

Furthermore, let $\varphi_{j} \in C_{0}^{\infty}\left(B(0,4) \times\left(0, \Lambda\left(T_{\text {pos }}\right)\right)\right)$ be a test function such that it vanishes on the parabolic boundary of $Q_{j}$ and $\varphi_{j}=1$ in $Q_{j+1}$. We may choose it so that

$$
\left|\nabla \varphi_{j}\right| \leq C 2^{j}, \quad\left(\frac{\partial \varphi_{j}}{\partial t}\right)_{+} \leq \frac{C 2^{j}}{T} \leq C 2^{j} k_{j}^{p-2} .
$$

We have the estimate

$$
\left(v-k_{j}\right)_{-}^{2} \geq \frac{\left(v-k_{j}\right)_{-}^{p}}{k_{j}^{p-2}} \geq \frac{T}{2}\left(v-k_{j}\right)_{-}^{p} .
$$

We obtain by Remark 2.3 that

$$
\begin{aligned}
& \frac{1}{T} \int_{Q_{j}}\left|\nabla\left(\left(v-k_{j}\right)_{-} \varphi_{j}\right)\right|^{p} d x d t+\underset{\Gamma_{j}}{\operatorname{ess} \sup } \int_{B_{j}}\left(v-k_{j}\right)_{-}^{p} \varphi_{j}^{p} d x \\
& \leq \frac{C}{T} \int_{Q_{j}}\left(v-k_{j}\right)_{-}^{p}\left|\nabla \varphi_{j}\right|^{p} d x d t+\frac{C}{T} \int_{Q_{j}}\left(v-k_{j}\right)_{-}^{2}\left(\frac{\partial \varphi_{j}^{p}}{\partial t}\right)_{+} d x d t \\
& \leq \frac{C 2^{p j}}{T} \int_{Q_{j}}\left(v-k_{j}\right)_{-}^{p}+\left(v-k_{j}\right)_{-}^{2} k_{j}^{p-2} d x d t
\end{aligned}
$$

A change of variables $z=t / T$ gives now

$$
\begin{aligned}
& \int_{T_{j} / T}^{4} \int_{B_{j}}\left|\nabla\left(\left(w-k_{j}\right)_{-} \psi_{j}\right)\right|^{p} d x d z+\underset{T_{j} / T<t<4}{\operatorname{ess} \sup _{B_{j}}} \int_{1}\left(w-k_{j}\right)_{-}^{p} \psi_{j}^{p} d x \\
& \leq C 2^{p j} \int_{T_{j} / T}^{4} \int_{B_{j}}\left(w-k_{j}\right)_{-}^{p}+\left(w-k_{j}\right)_{-}^{2} k_{j}^{p-2} d x d z,
\end{aligned}
$$

where $w(x, t)=v(x, T t)$ and $\psi_{j}(x, t)=\varphi_{j}(x, T t)$. Let now

$$
A_{j}=\int_{T_{j} / T}^{4} \int_{B_{j}} \chi_{\left\{w<k_{j}\right\}} d x d z=\frac{1}{T} \int_{T_{j}}^{4 T} \int_{B_{j}} \chi_{\left\{v<k_{j}\right\}} d x d t
$$

Note that by Lemma 3.7 we have the estimate $A_{0} \leq 3 v^{*}|B(0,3)|$ for the first level set. From the parabolic Sobolev's imbedding (see [8, Corollary 3.1, page 9]) and 
inequality (3.2), we get

$$
\begin{aligned}
& \int_{T_{j} / T}^{4} \int_{B_{j}}\left(w-k_{j}\right)_{-}^{p} \psi_{j}^{p} d x d z \\
& \leq C A_{j}^{p /(n+p)}\left(\int_{T_{j} / T}^{4} \int_{B_{j}}\left|\nabla\left(\left(w-k_{j}\right)_{-} \psi_{j}\right)\right|^{p} d x d z\right. \\
& \left.\quad+\underset{T_{j} / T<t<4}{\operatorname{ess} \sup _{B_{j}}} \int_{B_{j}}\left(w-k_{j}\right)_{-}^{p} \psi_{j}^{p} d x\right) \\
& \leq C 2^{p j} A_{j}^{1+p /(n+p)} k_{j}^{p} .
\end{aligned}
$$

Finally, we have

$$
\begin{aligned}
\int_{T_{j} / T}^{4} \int_{B_{j}}\left(w-k_{j}\right)_{-}^{p} \psi_{j}^{p} d x d z & \geq \int_{T_{j+1} / T}^{4} \int_{B_{j+1}}\left(w-k_{j}\right)_{-}^{p} d x d z \\
& \geq\left(k_{j}-k_{j+1}\right)^{p} A_{j+1} \geq \frac{k_{j}^{p}}{C 2^{p j}} A_{j+1} .
\end{aligned}
$$

This yields an iteration inequality

$$
A_{j+1} \leq C 4^{p j} A_{j}^{1+p /(n+p)} .
$$

By a standard argument (see [8, Lemma 4.1, page 12]) we have

$$
A_{j} \rightarrow 0, \quad \text { if } \quad A_{0} \leq C^{-(n+p) / p} 4^{-(n+p)^{2} / p} .
$$

By taking

$$
v^{*}=C^{-(n+p) / p} 4^{-(n+p)^{2} / p}(3|B(0,3)|)^{-1},
$$

we indeed have that

$$
v(x, t) \geq 2^{-M-1}
$$

for almost every $(x, t) \in B(0,4) \times(2 T, 4 T), T=2^{1+M(p-2)}$. Note that $v^{*}$ depends only on the structural constants. That is why also $M$ depends only on the structural constants. The result follows with

$$
T^{*}=\Lambda^{-1}(4 T)=\frac{\exp \left(\kappa(p-2) 2^{M(p-2)+3}\right)-1}{\kappa(p-2)}
$$

and

$$
\mu^{*}=2^{-M-1} \exp \left(-\kappa 2^{M(p-2)+3}\right) .
$$

Both constants are stable as $p \rightarrow 2$.

We are ready to prove the proposition on the expansion of positivity. 
Proof of Proposition 3.1. We introduce the scaled function

$$
v(x, t)=u\left(x_{0}+R x, t_{0}+R^{p} t\right) .
$$

It is a weak supersolution in a neighborhood of $B\left(0,4 R_{0} / R\right) \times\left(0, R^{-p} T_{0}\right)$. For the Lebesgue instant 0 , we have

$$
|\{x \in B(0,1): v(x, 0)>N\}| \geq \delta|B(0,1)| .
$$

We may replace $\delta$ by $2^{-n} \delta$, if necessary, and assume that $R_{0} / R=2^{I}$ for some $I \in \mathbb{N}$. Let $\mu^{*}$ and $T^{*}$ be as in Lemma 3.8 corresponding $\delta$. It follows that

$$
\underset{Q}{\operatorname{essinf}} v \geq \mu^{*}
$$

where $Q=B(0,2) \times\left(N^{2-p} T^{*} / 2, N^{2-p} T^{*}\right)$, provided that $N^{2-p} T^{*} \leq R^{-p} T_{0}$. Let then $q \leq I, q \in \mathbb{N}$. We define $N_{q}=\left(\mu^{*}\right)^{q} N$ and $R_{q}=2^{q} R$. Suppose now that for some Lebesgue instant $T_{q}$ we have

$$
u\left(x, N^{2-p} T_{q}\right) \geq N \mu_{q}, \quad x \in B\left(x_{0}, 2^{q} R\right) .
$$

We scale this supersolution as

$$
v_{q}(x, t)=u\left(x_{0}+2^{q} R x, t_{0}+T_{q}+2^{p q} R^{p} t\right) .
$$

Then also $v_{q}$ is a supersolution in an open set which compactly contains the set $B\left(0,2^{-q+2} R_{0} / R\right) \times\left(0,2^{-p q} R^{-p} T_{0}-T_{q}\right)$, provided that $2^{-p q} R^{-p} T_{0}>T_{q}$, Moreover, $v_{q}(x, 0) \geq N_{q}$ for $x \in B(0,1)$. Since 0 is a Lebesgue instant for $v_{q}$, Lemma 3.8 implies

$$
\underset{Q_{q}}{\operatorname{ess} \inf } v_{q} \geq N_{q} \mu^{*}=N_{q+1},
$$

where $Q_{q}=B(0,2) \times\left(N_{q}^{2-p} T^{*} / 2, N_{q}^{2-p} T^{*}\right)$. We may pick $T_{q+1}$ from the interval $\left(N_{q}^{2-p} T^{*} / 2, N_{q}^{2-p} T^{*}\right)$ such that

$$
T_{q+1}=T_{q}+\frac{T^{*}}{2}\left(N_{q}^{2-p} 2^{p q} R^{p}+\frac{N^{2-p} R^{p}}{2^{p}\left(\mu^{*}\right)^{2-p}-1}\right),
$$

and, without losing the generality, we may assume that $T_{q+1}$ is a Lebesgue instant. We obtain the formula

$$
T_{q}=t_{0}+C N_{q}^{2-p} 2^{p q} R^{p} .
$$

An induction argument implies that for $\tilde{\theta}=-\log _{2} \mu^{*}>0$ there is a Lebesgue instant

such that

$$
\widetilde{T}=C\left(N\left(R / R_{0}\right)^{\widetilde{\theta}}\right)^{2-p} R_{0}^{p}
$$

$$
\underset{x \in B\left(x_{0}, R_{0}\right)}{\operatorname{essinf}} u(x, \widetilde{T}) \geq N\left(R / R_{0}\right)^{\tilde{\theta}} .
$$

Finally, we scale

$$
v(x, t)=u\left(x_{0}+R_{0} x, t_{0}+\widetilde{T}+R_{0}^{p} t\right),
$$

and apply once more Lemma 3.8, which completes the proof. 


\section{The hot alternative}

We now proceed to the analysis of the hot alternative. The main result of the section is the following lemma.

Lemma 4.1. There are positive constants $\sigma, T_{h}$ and $\vartheta_{h}$, all depending only on $n$, $p, \mathcal{A}_{0}$, and $\mathcal{A}_{1}$, such that if $u$ is a supersolution in $B(0,8) \times\left(0,2 T_{h}\right)$ and

$$
\left|\left\{x \in B(0,2): u\left(x, t_{0}\right)>8 k^{1+\sigma}\right\}\right|>8 k^{-\sigma}|B(0,2)|
$$

for some $k>8^{1 / \sigma}$ and some Lebesgue instant $0<t_{0}<2^{p}$, then

$$
\underset{B(0,2) \times\left(T_{h}, 2 T_{h}\right)}{\operatorname{ess} \inf } u \geq \vartheta_{h} .
$$

We first explain the strategy of the proof. The assumed positivity (4.1) can be carried further in time with the aid of Lemma 3.2. We then cover the obtained positivity set using a refined Krylov-Safonov covering argument. It takes into account two different possibilities: either the level set is porous or it is concentrated. In both cases, an application of Proposition 3.1 leads to the quantitative increment of the level set, but with a smaller level. By porous we mean that we can cover the level set with small space-time cylinders such that by multiplying the radii of them by three, the measure of the union of expanded cylinders is, say four times, larger than the measure of the original level set. An iteration argument then implies the result of Lemma 4.1. The dominant phenomenon is that the expansion of positivity occurs only after a waiting time which depends on the spatial scale. The covering argument gives different scales and a delicate part of the proof is to glue these together.

We define the following scaled space-time cylinder

$$
U^{\zeta}((x, t), R)=B(x, R) \times\left(t-\zeta^{2-p} R^{p}, t+\zeta^{2-p} R^{p}\right)
$$

where $\zeta>0$, and the metric

$$
d_{U}((x, t),(y, s))=\max \left\{|x-y|, \zeta^{(p-2) / p}|t-s|^{1 / p}\right\}
$$

We note that $U^{\zeta}$ is a ball with respect to this metric, i.e.

$$
U^{\zeta}(z, R)=\left\{y \in \mathbb{R}^{n} \times \mathbb{R}: d_{U}(z, y)<R\right\} .
$$

For the brevity, we denote $5 U=U^{\zeta}(z, 5 R)$. The doubling property allows us to use the countable version of Vitali's covering theorem, which states that if $E$ is a bounded set in $\mathbb{R}^{n+1}$ and $\mathcal{F}$ is a collection of space-time cylinders $U^{\zeta}$ intersecting and covering $E$, then there is a countable and pairwise disjoint subcollection $\mathcal{G}$ of $\mathcal{F}$ such that

$$
E \subset \bigcup_{U \in \mathcal{G}} 5 U .
$$

For the proof, see for example [16]. 
Let now $E$ be a measurable subset of $F$ in $\mathbb{R}^{n} \times \mathbb{R}$. We define the set

$$
\Psi_{\delta}^{\rho, \zeta}(E, F)=\bigcup_{0<r<\rho}\left\{U^{\zeta}(z, 3 r) \cap F: z \in F,\left|U^{\zeta}(z, 3 r) \cap E\right|>\delta\left|U^{\zeta}(z, r)\right|\right\},
$$

where $\rho>0$ and $0<\delta<1$. The parameter $\rho$ is a threshold radius for the size of the space-time cylinders. It measures the before described porosity of the level set and it will be used to control the waiting time in the iteration scheme.

The standard version of the following refined Krylov-Safonov covering argument is without any threshold radius $\rho$. The proof follows [19].

Lemma 4.2. Let $E$ be a measurable subset of $Q_{0}=B\left(x_{0}, R_{0}\right) \times\left(t_{0}, t_{0}+T_{0}\right)$. Then at least one of the following three cases holds:

(1) $|E| \leq \delta 2^{n+p}\left|\Psi_{\delta}^{\rho, \zeta}\left(E, Q_{0}\right)\right|$,

(2) there is $z \in Q_{0}$ such that $\left|U^{\zeta}(z, \rho) \cap E\right|>60^{-n-p} \delta\left|U^{\zeta}(z, \rho)\right|$,

(3) $\Psi_{\delta}^{\rho, \zeta}\left(E, Q_{0}\right)=Q_{0}$ and $|E|>15^{-n-p} \delta\left|Q_{0}\right|$.

Proof. In the proof we use the abbreviation

$$
U(z, r)=U^{\zeta}(z, r) \quad \text { and } \quad \Psi_{\delta}=\Psi_{\delta}^{\rho, \zeta}\left(E, Q_{0}\right) .
$$

We first define the maximal operator $M: \mathbb{R}^{n} \times \mathbb{R} \mapsto \mathbb{R}$ as

$$
M(z)=\sup \frac{|E \cap U(y, 3 r)|}{|U(y, r)|},
$$

where the supremum is taken over all space-time cylinders $U(y, 3 r)$, such that $y \in$ $Q_{0}, 0<r<\rho$ and $z \in U(y, 3 r)$. We claim that

$$
\Psi_{\delta}=\left\{z \in Q_{0}: M(z)>\delta\right\} .
$$

Let first $z \in Q_{0}$ be such that $M(z)>\delta$. Then there is a cylinder $U(y, 3 r)$, such that $y \in Q_{0}, 0<r<\rho,|E \cap U(y, 3 r)|>\delta|U(y, r)|$ and $z \in U(y, 3 r)$. This means that $z \in \Psi_{\delta}$. For the converse inclusion, if $z \in \Psi_{\delta}$, then there is a cylinder $U(y, 3 r)$, $y \in Q_{0}$ and $0<r<\rho$, such that $|E \cap U(y, 3 r)|>\delta|U(y, r)|$ and $z \in U(y, 3 r)$. This implies that $M(z)>\delta$.

Suppose that $Q_{0} \backslash \Psi_{\delta} \neq \emptyset$. The set $\Psi_{\delta}$ is open by definition and, consequently, we have for every $z \in \Psi_{\delta}$ that

$$
r_{z}=\frac{1}{2} \sup \left\{r>0: z \in U(y, r) \subset \Psi_{\delta}, U(y, 2 r) \cap\left(Q_{0} \backslash \Psi_{\delta}\right)=\emptyset\right\}
$$

is a positive number. Let us first assume that every $r_{z}<\rho / 4$. For each $z \in \Psi_{\delta}$ we find $y_{z}$ such that $z \in U\left(y_{z}, r_{z}\right) \subset \Psi_{\delta}$ and $U\left(y_{z}, 5 r_{z}\right) \cap\left(Q_{0} \backslash \Psi_{\delta}\right) \neq \emptyset$. Clearly the union of $U\left(y_{z}, r_{z}\right)$ covers $\Psi_{\delta}$. By Vitali's covering theorem, there are countably 
many pairwise disjoint space-time cylinders $U\left(y_{i}, r_{i}\right)$, where $y_{i}=y_{z_{i}}$ and $r_{i}=r_{z_{i}}$, $i=1,2, \ldots$, such that

$$
\Psi_{\delta} \subset \bigcup U\left(y_{i}, 5 r_{i}\right) .
$$

By the construction we have $U\left(y_{i}, 5 r_{i}\right) \cap\left(Q_{0} \backslash \Psi_{\delta}\right) \neq \emptyset$ for every $i=1,2, \ldots$, and there is a point $\eta_{i} \in U\left(y_{i}, 5 r_{i}\right) \cap\left(Q_{0} \backslash \Psi_{\delta}\right)$. In particular, $M\left(\eta_{i}\right) \leq \delta, i=1,2, \ldots$ Since $\eta_{i} \in U\left(y_{i}, 5 r_{i}\right)$ and $5 r_{i} / 3<\rho$, we conclude that

$$
\left|E \cap U\left(y_{i}, 5 r_{i}\right)\right| \leq \delta\left|U\left(y_{i}, \frac{5 r_{i}}{3}\right)\right| \leq 2^{n+p} \delta\left|U\left(y_{i}, r_{i}\right)\right| .
$$

Furthermore, if $z$ is a density point of $E$, then

$$
\liminf _{r \rightarrow 0} \frac{|E \cap U(z, 3 r)|}{|U(z, r)|} \geq \liminf _{r \rightarrow 0} \frac{|E \cap U(z, r)|}{|U(z, r)|}=1>\delta .
$$

Almost every point of $E$ is a density point. Hence we obtain that almost every point of $E$ belongs to $\Psi_{\delta}$. From this it follows that

$$
|E|=\left|E \cap \Psi_{\delta}\right| \leq \sum\left|E \cap U\left(y_{i}, 5 r_{i}\right)\right| \leq 2^{n+p} \delta \sum\left|U\left(y_{i}, r_{i}\right)\right| \leq 2^{n+p} \delta\left|\Psi_{\delta}\right| .
$$

We are left with the cases that $\Psi_{\delta}$ contains a space-time cylinder $U(y, \rho / 4)$ or $\Psi_{\delta}=Q_{0}$. If $U(y, \rho / 4) \subset \Psi_{\delta}$, then the definition of $\Psi_{\delta}$ implies that for all $\eta \in$ $U(y, \rho / 4)$, we find $z_{\eta} \in Q_{0}$ and $0<r_{\eta}<\rho$ such that

$$
\left|U\left(z_{\eta}, 3 r_{\eta}\right) \cap E\right|>\delta\left|U\left(z_{\eta}, r_{\eta}\right)\right|
$$

and $U(y, \rho / 4) \cap U\left(z_{\eta}, 3 r_{\eta}\right) \neq \emptyset$. We first suppose that $r_{\tilde{\eta}} \geq \rho / 8$ for some $\tilde{\eta}$. We cover $U\left(z_{\tilde{\eta}}, 3 r_{\tilde{\eta}}\right)$ with $5^{n+p}$ space-time cylinders, all with radius $\rho$. Then, by the pigeonhole principle, for at least one of these cylinders, say $U(z, \rho)$, must hold

$$
|U(z, \rho) \cap E|>5^{-n-p} \delta|U(z, \rho / 8)|,
$$

and the result follows in this case. Suppose then that for all $\eta$ we have $r_{\eta}<\rho / 8$. Then $\cup_{\eta} U\left(z_{\eta}, 3 r_{\eta}\right) \subset U(y, \rho)$. By Vitali's covering theorem we find a set $A \subset$ $U(y, \rho / 4)$ such that the collection of space-time cylinders $\left\{U\left(z_{\eta}, 3 r_{\eta}\right)\right\}$ are pairwise disjoint for all $\eta \in A$ and

$$
U(y, \rho / 4) \subset \bigcup_{\eta \in A} U\left(z_{\eta}, 15 r_{\eta}\right) .
$$

Thus we obtain

$$
\begin{aligned}
|U(y, \rho) \cap E| & \geq \sum_{\eta \in A}\left|U\left(z_{\eta}, 3 r_{\eta}\right) \cap E\right| \\
& >\delta \sum_{\eta \in A}\left|U\left(z_{\eta}, r_{\eta}\right)\right| \\
& =15^{-n-p} \delta \sum_{\eta \in A}\left|U\left(z_{\eta}, 15 r_{\eta}\right)\right| \\
& \geq 15^{-n-p} \delta|U(y, \rho / 4)| \\
& =60^{-n-p} \delta|U(y, \rho)| .
\end{aligned}
$$


This is the second case of the lemma.

If $\Psi_{\delta}=Q_{0}$, then by Vitali's covering theorem we have countable subset $\left(z_{i}\right)$ of $Q_{0}$ such that the family $\left\{U\left(z_{i}, 3 r_{i}\right)\right\}$ is pairwise disjoint, $Q_{0} \subset \cup U\left(z_{i}, 15 r_{i}\right)$ and

$$
\left|U\left(z_{i}, 3 r_{i}\right) \cap E\right|>\delta\left|U\left(z_{i}, r_{i}\right)\right| .
$$

Therefore, we conclude

$$
\begin{aligned}
|E| & \geq \sum\left|U\left(z_{i}, 3 r_{i}\right) \cap E\right| \\
& >\delta \sum\left|U\left(z_{i}, r_{i}\right)\right| \\
& =15^{-n-p} \delta \sum\left|U\left(z_{i}, 15 r_{i}\right)\right| \\
& \geq 15^{-n-p} \delta\left|Q_{0}\right| .
\end{aligned}
$$

This completes the proof.

We now analyze the different cases emerging from Lemma 4.2. The first case gives the following result.

Proposition 4.3. Let $N>0, Q_{0}=B\left(x_{0}, R_{0}\right) \times\left(t_{0}, t_{0}+T_{0}\right), 0<\rho<R_{0}$ and $0<\delta<1$. There are constants $C_{i}=C_{i}\left(n, p, \mathcal{A}_{0}, \mathcal{A}_{1}, \delta\right) \geq 2, i=1$, 2, such that if $u$ is a weak supersolution in $B\left(x_{0}, 12 R_{0}\right) \times\left(t_{0}, t_{0}+T_{1}\right), T_{1}=T_{0}+C_{2} N^{2-p} \rho^{p}$, and for $E=\left\{(x, t) \in Q_{0}: u(x, t)>N\right\}$ we have

$$
\left|\Psi_{\delta}^{\rho, N}\left(E, Q_{0}\right)\right| \geq 4|E|
$$

then

$$
\left|\left\{(x, t) \in Q: u(x, t) \geq \frac{1}{C_{1}} N\right\}\right| \geq 2|E|,
$$

where $Q=B\left(x_{0}, R_{0}\right) \times\left(t_{0}, t_{0}+T_{1}\right)$.

The goal is to reduce the proof of Proposition 4.3 to the following elementary one dimensional argument with the aid of Fubini's theorem.

Proposition 4.4. Let $\left\{I_{j}\right\}, I_{j}=\left(\alpha_{j}, \beta_{j}\right)$, be a finite family of intervals in $\mathbb{R}, j=$ $1,2, \ldots, J$, and let $f_{0}=0$. Let $f_{j}: \mathbb{R} \mapsto\{-1,0,1\}$ be

$$
f_{j}(x)= \begin{cases}-1, & x \in I_{j} \\ 1, & x \in\left(\beta_{j}+M\left(\beta_{j}-\alpha_{j}\right), \beta_{j}+3 M\left(\beta_{j}-\alpha_{j}\right)\right), \\ 0 & \text { otherwise }\end{cases}
$$

where $M \geq 1, j=1,2, \ldots, J$. Then

$$
\left|\left\{x \in \mathbb{R}: \sup _{j} f_{j}(x)=1\right\}\right| \geq \frac{2 M}{3 M+1}\left|\left\{x \in \mathbb{R}: \inf _{j} f_{j}(x)=-1\right\}\right| .
$$


Proof. We first collect maximal intervals, say $J_{i}^{+}=\left(a_{i}, b_{i}\right)$, from the set

$$
D^{+}=\left\{x \in \mathbb{R}: \sup _{j} f_{j}(x)=1\right\} .
$$

By the maximal we mean that for any $\varepsilon>0$ neither $\left(a_{i}-\varepsilon, b_{i}\right)$ nor $\left(a_{i}, b_{i}+\varepsilon\right)$ belongs to $D^{+}$. Assume then that for some $i$ the set $\left\{x \in \mathbb{R}: f_{i}(x)=1\right\}$ intersects $J_{j}^{+}$. The maximality of $J_{j}^{+}$implies that the whole set $\left\{x \in \mathbb{R}: f_{i}(x)=1\right\}$ belongs to $J_{j}^{+}$and, hence, the support of $f_{i}$ belongs to $J_{j}^{-}=\left(c_{j}, b_{j}\right)$, where $c_{j}=a_{j}-\left(b_{j}-a_{j}\right)(M+1) /(2 M)$.

Next we define the function

$$
f(x)=\sup f_{j}(x)+\inf f_{j}(x)
$$

and claim that

$$
\int_{\mathbb{R}} f(x) d x \geq-\frac{M+1}{2 M}\left|\left\{x \in \mathbb{R}: \sup f_{j}(x)=1\right\}\right| .
$$

This will prove the result since

$$
\int_{\mathbb{R}} f(x) d x=\left|\left\{x \in \mathbb{R}: \sup f_{j}(x)=1\right\}\right|-\left|\left\{x \in \mathbb{R}: \inf f_{j}(x)=-1\right\}\right| .
$$

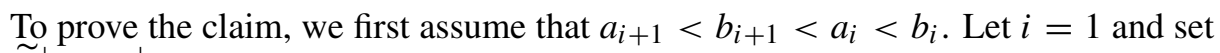
$\widetilde{J}_{1}^{+}=J_{1}^{+}$. We define the sets

$$
\begin{aligned}
& \Lambda_{i}^{1}=\left\{k: b_{k}<\widetilde{a}_{i}, J_{k}^{+} \subset \widetilde{J}_{i}^{-}, c_{k} \geq \widetilde{c}_{i}\right\}, \\
& \Lambda_{i}^{2}=\left\{k: b_{k}<\widetilde{a}_{i}, J_{k}^{+} \subset \widetilde{J}_{i}^{-}, c_{k}<\widetilde{c}_{i}\right\}, \\
& \Lambda_{i}^{3}=\left\{k: a_{k}<\widetilde{c}_{i}<b_{k}\right\}, \\
& \Lambda_{i}^{4}=\left\{k: b_{k}<\widetilde{c}_{i}\right\}
\end{aligned}
$$

and choose

$$
k=\min \left\{\Lambda_{i}^{2} \cup \Lambda_{i}^{3} \cup \Lambda_{i}^{4}\right\},
$$

provided that the set on the right-hand side is non-empty. We define $\widetilde{\sim}_{i+1}=a_{k}$, $b_{i+1}=b_{k}, \widetilde{c}_{i+1}=c_{k}$ and denote $\widetilde{J}_{i+1}^{+}=J_{k}^{+}, \widetilde{J}_{i+1}^{+}=\left(\widetilde{a}_{i+1}, \widetilde{b}_{i+1}\right)$. Note that we ignore all the intervals $J_{k}^{+}, k \in \Lambda_{i}^{1}$. By doing so we estimate the integral of $f$ below. We repeat the reasoning above for $i=1,2, \ldots$, and stop when the set $\Lambda_{i}^{2} \cup \Lambda_{i}^{3} \cup \Lambda_{i}^{4}$ is empty for the first time. Say that this happens when $i=I$. We rewrite

$$
\int_{\mathbb{R}} f(x) d x=\int_{\widetilde{c}_{I}}^{\widetilde{b}_{I}} f(x) d x+\sum_{i=1}^{I-1} \int_{\widetilde{b}_{i+1}}^{\widetilde{b}_{i}} f(x) d x .
$$

First, since $\sup _{j} f_{j}(x)=1$ in $\left(\widetilde{a}_{I}, \tilde{b}_{I}\right)$, we have

$$
\int_{\widetilde{c}_{I}}^{\widetilde{b}_{I}} f(x) d x \geq \int_{\widetilde{c}_{I}}^{\tilde{a}_{I}} f(x) d x \geq-\left(\widetilde{a}_{I}-\widetilde{c}_{I}\right)
$$


Next, if the index $k$ corresponding to the set $\widetilde{J}_{i+1}^{+}$belongs to $\Lambda_{i}^{2}$ or $\Lambda_{i}^{3}$, then we have

$$
\int_{\widetilde{b}_{i+1}}^{\widetilde{b}_{i}} f(x) d x \geq \int_{\widetilde{b}_{i+1}}^{\tilde{a}_{i}} f(x) d x \geq-\left(\widetilde{a}_{i}-\widetilde{b}_{i+1}\right) \geq-\left(\widetilde{a}_{i}-\widetilde{c}_{i}\right) .
$$

If $k$ is in $\Lambda_{i}^{4}$, then $f(x)=0$ in $\left(\tilde{b}_{i+1}, \widetilde{c}_{i}\right)$. Indeed, if $f(x)=-1$ for some $x$ in $\left(\tilde{b}_{i+1}, \widetilde{c}_{i}\right)$, then there would be $j<k$ such that $\widetilde{b}_{i+1}<c_{j}<\widetilde{c}_{i}$, and, consequently, $j$ would belong to $\Lambda_{i}^{2}$ or $\Lambda_{i}^{3}$. This contradicts the choice of $k$. Therefore, we have

$$
\int_{\widetilde{b}_{i+1}}^{\widetilde{b}_{i}} f(x) d x \geq \int_{\widetilde{c}_{i}}^{\widetilde{a}_{i}} f(x) d x \geq-\left(\widetilde{a}_{i}-\widetilde{c}_{i}\right) .
$$

We conclude that

$$
\int_{\mathbb{R}} f(x) d x \geq-\sum\left(\tilde{a}_{i}-\tilde{c}_{i}\right)=-\frac{M+1}{2 M} \sum\left(\tilde{b}_{i}-\tilde{a}_{i}\right) \geq-\frac{M+1}{2 M}\left|D^{+}\right|,
$$

which proves the claim.

As was noted before, the positivity expands after a certain waiting time. Therefore, even the space-time cylinders in $\Psi_{\delta}$ would not overlap, the expanded cylinders might very well do so. The previous proposition then gives the control on the overlap.

Proof of Proposition 4.3. Let $\varepsilon>0$. We take a finite collection of cylinders $U^{N}\left(z_{j}, r_{j}\right), z_{j}=\left(x_{j}, t_{j}\right) \in Q_{0}$ and $r_{j}<\rho / 3$ such that

$$
\left(U^{N}\left(z_{j}, r_{j}\right) \cap Q_{0}\right) \subset \Psi_{\delta}^{\rho, N}\left(E, Q_{0}\right), \quad i=1,2, \ldots, J,
$$

and

$$
\left|\left(\cup_{j} U_{j}^{N}\left(z_{j}, r_{j}\right)\right) \cap Q_{0}\right| \geq\left|\Psi_{\delta}^{\rho, N}\left(E, Q_{0}\right)\right|-\varepsilon
$$

By the definition of $\Psi_{\delta}^{\rho, N}$, we have

$$
\left|E \cap U^{N}\left(z_{j}, r_{j}\right)\right|>\delta 3^{-n-p}\left|U^{N}\left(z_{j}, r_{j}\right)\right| .
$$

It follows that there is a Lebesgue instant $t_{j}-N^{2-p} r_{j}^{p}<\bar{t}_{j}<t_{j}+N^{2-p_{r}} r_{j}^{p}$ such that

$$
\left|\left\{x \in B\left(x_{j}, r_{j}\right): u\left(x, \bar{t}_{j}\right)>N\right\}\right|>\delta 3^{-n-p}\left|B\left(x_{j}, r_{j}\right)\right| .
$$

We then apply Proposition 3.1 and obtain that there are constants as in the statement such that

$$
\underset{Q_{j}}{\operatorname{essinf}} u \geq \frac{1}{C_{1}} N
$$


where $Q_{j}=B\left(x_{j}, 3 r_{j}\right) \times\left(\bar{t}_{j}+C_{2} N^{2-p} r_{j}^{p}, \bar{t}_{j}+3 C_{2} N^{2-p} r_{j}^{p}\right)$. Note that we have

$$
t_{0}<\bar{t}_{j}+C_{2} N^{2-p} r_{j}^{p}<\bar{t}_{j}+3 C_{2} N^{2-p} r_{j}^{p}<t_{0}+T_{0}+C_{2} N^{2-p} \rho^{p} .
$$

Furthermore, we define functions $f_{j}: \mathbb{R}^{n} \times \mathbb{R} \mapsto\{-1,0,1\}$ as

$$
f_{j}(x, t)= \begin{cases}-1, & (x, t) \in U^{N}\left(z_{j}, r_{j}\right) \cap Q_{0}, \\ 1, & (x, t) \in Q_{j}, \\ 0, & \text { otherwise, }\end{cases}
$$

$i=1,2, \ldots, J$, and $f_{0}=0$. It readily follows from Proposition 4.4 that we have

$$
\left|\left\{t \in \mathbb{R}: \sup _{j} f_{j}(x, t)=1\right\}\right| \geq \frac{1}{2}\left|\left\{t \in \mathbb{R}: \inf _{j} f_{j}(x, t)=-1\right\}\right|
$$

for every $x \in B\left(x_{0}, R_{0}\right)$. By integrating both sides over the ball $B\left(x_{0}, R_{0}\right)$, we conclude

$$
\left|\left\{(x, t) \in Q: u(x, t) \geq \frac{1}{C_{1}} N\right\}\right| \geq \frac{1}{2}\left|\left(\cup U_{j}^{N}\left(z_{j}, r_{j}\right)\right) \cap Q_{0}\right|,
$$

where

$$
Q=B\left(x_{0}, R_{0}\right) \times\left(t_{0}, t_{0}+T_{0}+C_{2} N^{2-p} \rho^{p}\right) .
$$

Since $\varepsilon$ is arbitrary, the result follows.

To prove Lemma 4.1, we use refined Krylov-Safonov covering Lemma 4.2 together with the expansion of positivity, Proposition 3.1. The idea is to use subsequently Lemma 4.2. At each step, by decreasing the level $N$ of the level set, we obtain an increment in the measure of the new level set. It is evident by Proposition 4.3 that when $N$ is small, we need to choose the threshold radius $\rho$ small in order to control the stretching of the space-time cylinders in the time direction. However, we may end up to the second case in Lemma 4.2, which states that the level set intersects a large proportion of a space-time cylinder with the side length comparable to the small threshold radius. Nevertheless, we may tailor the choice of $\sigma$ in Lemma 4.1 so that the desired result follows directly from the expansion of positivity in this case.

Proof of Lemma 4.1. Let $C_{1}$ and $C_{2}$ be as in Proposition 4.3 with $\delta=2^{-n-p-2}$. To begin with, we take an initial step by using Lemma 3.2 and obtain that

$$
|\{x \in B(0,2): u(x, t)>k\}| \geq k^{-\sigma}|B(0,2)|
$$

for almost every

$$
t_{0}<t<t_{0}+\frac{1}{C_{3}} k^{-\sigma(p+1)} k^{(2-p)(1+\sigma)}=t_{0}+\frac{1}{C_{3}} k^{2-p} k^{(1-2 p) \sigma} .
$$


Let treshold radii in Lemma 4.2 be

$$
\rho_{1}=\left(\frac{1}{C_{2} C_{3}} k^{(1-2 p) \sigma}\right)^{1 / p}, \quad \rho_{i}=\rho_{1}\left(2 C_{1}^{p-2}\right)^{(1-i) / p}, \quad i=2,3, \ldots
$$

We define

$$
Q_{1}=B(0,2) \times\left(t_{1}, t_{1}+\frac{C_{2}}{2} k^{2-p} \rho_{1}^{p}\right), \quad t_{1}=t_{0}+\frac{C_{2}}{2} k^{2-p} \rho_{1}^{p} .
$$

This is the initial set for the iteration scheme. Integrating (4.2) over the interval $\left(t_{1}, t_{1}+C_{2} k^{2-p} \rho_{1}^{p} / 2\right)$ gives us

$$
\left|\left\{(x, t) \in Q_{1}: u(x, t)>k\right\}\right| \geq k^{-\sigma}\left|Q_{1}\right| .
$$

Furthermore, we set

$$
k_{i}=k C_{1}^{1-i} \quad \text { and } \quad t_{i+1}=t_{i}+C_{2} k_{i}^{2-p} \rho_{i}^{p}=t_{i}+C_{2} k^{2-p} \rho_{1}^{p} 2^{1-i},
$$

$i=1,2, \ldots$, and define

$$
Q_{i}=B(0,2) \times\left(t_{1}, t_{i}\right), \quad i=2,3, \ldots
$$

A straightforward calculation gives

$$
t_{i}-t_{1}=C_{2} k^{2-p} \rho_{1}^{p} \sum_{j=0}^{i-2} 2^{-j}=C_{2} k^{2-p} \rho_{1}^{p} \frac{1-2^{1-i}}{1-2^{-1}}, \quad i=2,3, \ldots,
$$

and, hence,

$$
\lim _{i \rightarrow \infty}\left|Q_{i}\right|=4\left|Q_{1}\right|
$$

We denote

$$
E_{i}=\left\{(x, t) \in Q_{i}: u(x, t)>k_{i}\right\} .
$$

An application of Lemma 4.2 with the set $E_{i}$ and $\delta=2^{-n-p-2}$ gives that at least one of the following holds

$$
\begin{gathered}
\left|\Psi_{\delta}^{\rho_{i}, k_{i}}\left(E_{i}, Q_{i}\right)\right| \geq 4\left|E_{i}\right|, \\
\left|U^{k_{i}}\left(z, \rho_{i}\right) \cap E_{i}\right|>120^{-n-p-2}\left|U^{k_{i}}\left(z, \rho_{i}\right)\right|, \quad z \in Q_{i}, \\
\left|E_{i}\right|>30^{-n-p-2}\left|Q_{i}\right| .
\end{gathered}
$$

From (4.5) it follows by the definitions of $k_{i+1}$ and $t_{i+1}$, and Proposition 4.3 that

$$
\left|\left\{(x, t) \in Q_{i+1}: u(x, t)>k_{i+1}\right\}\right| \geq 2\left|\left\{(x, t) \in Q_{i}: u(x, t)>k_{i}\right\}\right| .
$$


If this happens for $j$ times, then

$$
\left|\left\{(x, t) \in Q_{j+1}: u(x, t)>k_{j+1}\right\}\right| \geq 2^{j}\left|\left\{(x, t) \in Q_{1}: u(x, t)>k\right\}\right| .
$$

Therefore, we find the integer $I$ such that

$$
2^{I-1} \leq k^{\sigma} \leq 2^{I} .
$$

The number $I$ defines the stopping time for our iteration argument. If we end up to (4.5) successively $I-1$ times, then by (4.3) and (4.4), we obtain

$$
\begin{aligned}
\left|\left\{(x, t) \in Q_{I}: u(x, t)>k_{I}\right\}\right| & \geq 2^{I-1}\left|\left\{(x, t) \in Q_{1}: u(x, t)>k\right\}\right| \\
& \geq 2^{I-1} k^{-\sigma}\left|Q_{1}\right| \\
& \geq \frac{1}{8}\left|Q_{I}\right| .
\end{aligned}
$$

Thus we find a Lebesgue instant $t_{1}<\bar{t}_{I}<t_{I}$ such that

$$
\left|\left\{x \in B(0,2): u\left(x, \bar{t}_{I}\right)>k_{I}\right\}\right| \geq \frac{1}{8}|B(0,2)| .
$$

We assume that $\sigma \leq 1 / \log _{2} C_{1}$ and, hence,

$$
k_{I}=k C_{1}^{1-I}=k C_{1} 2^{-I \log _{2} C_{1}} \geq \frac{1}{C_{4}} k^{1-\sigma \log _{2} C_{1}} \geq \frac{1}{C_{4}} .
$$

Proposition 3.1 yields the result in this case.

Suppose then that (4.6) realizes for some $j \leq I$. Then there is $z=(\bar{x}, \bar{t}) \in Q_{j}$ such that

$$
\left|\left\{(x, t) \in U^{\zeta_{j}}\left(z, \rho_{j}\right): u(x, t)>k_{j}\right\}\right|>120^{-n-p-2}\left|U^{\zeta_{j}}\left(z, \rho_{j}\right)\right| .
$$

The inequality implies that there is a Lebesgue instant $t_{1}<\tilde{t}<t_{j}$ such that

$$
\left|\left\{x \in B\left(\bar{x}, \rho_{j}\right): u(x, \widetilde{t})>k_{j}\right\}\right|>120^{-n-p-2} \mid B\left(\bar{x}, \rho_{j} \mid .\right.
$$

Since both radii $\rho_{j}=\rho_{1} C_{1}^{(1-j)(p-2) / p} 2^{(1-j) / p}$ and levels $k_{j}=C_{1}^{1-j} k$ are decreasing with respect to $j$, it is enough to check only the case $j=I$. We apply Proposition 3.1 and obtain with some positive constants $\bar{T}=\bar{T}\left(n, p, \mathcal{A}_{0}, \mathcal{A}_{1}\right)$ and $\theta=\theta\left(n, p, \mathcal{A}_{0}, \mathcal{A}_{1}\right)$ that

$$
\underset{U}{\operatorname{essinf}} u \geq k_{I} \rho_{I}^{\theta}
$$

where $U=B(0,2) \times(\bar{t}+T / 2, \bar{t}+T)$ and $T=\bar{T}\left(k_{I} \rho_{I}^{\theta}\right)^{2-p}$. Thus we have to calculate $\rho_{I}$ by means of $k$. Since

$$
\rho_{1}=\left(\frac{1}{C_{2} C_{3}} k^{(1-2 p) \sigma}\right)^{1 / p}
$$


we arrive at

$$
\rho_{I}=\rho_{1}\left(2 C_{1}^{p-2}\right)^{(1-I) / p}=\frac{1}{C_{5}} k^{(1-2 p) \sigma / p-\sigma \log _{2}\left(2 C_{1}^{p-2}\right) / p}=\frac{1}{C_{5}} k^{-\sigma C_{6}} .
$$

We conclude that

$$
\underset{U}{\operatorname{ess} \inf } u \geq \frac{1}{C_{7}} k^{1-\sigma\left(\log _{2} C_{1}+\theta C_{6}\right)}
$$

and $T=C_{8} k^{(2-p)\left(1-\sigma\left(\log _{2} C_{1}+\theta C_{6}\right)\right)}$. We set

$$
\sigma=\frac{1}{\log _{2} C_{1}+\theta C_{6}}
$$

which leads to the claim, again by Proposition 3.1.

We are left with the case (4.7). A similar reasoning as in the case of (4.8) implies the desired estimate and finishes the proof.

\section{The cold alternative}

The alternative to the hot domain is that the domain is cold. This means that (4.1) does not occur for any Lebesgue instant and for any large $k$. We have the following lemma.

Lemma 5.1. Let $\sigma=\sigma\left(n, p, \mathcal{A}_{0}, \mathcal{A}_{1}\right)$ be as in Lemma 4.1. There are positive constants $T_{c}, M_{c}$ and $\vartheta_{c}$, all depending only on $n, p, \mathcal{A}_{0}$, and $\mathcal{A}_{1}$, such that if $u$ is a weak supersolution in an open set which compactly contains $B(0,8) \times\left(0,2 T_{c}\right)$, 0 is a Lebesgue instant,

$$
f_{B(0,1)} u(x, 0) d x \geq M_{c}
$$

and

$$
\left|\left\{x \in B(0,2): u(x, t)>8 k^{1+\sigma}\right\}\right| \leq 8 k^{-\sigma}|B(0,2)|
$$

for every $k \geq 8^{1 / \sigma}$ and for almost every $0<t<2^{p}$, then

$$
\underset{B(0,2) \times\left(T_{c}, 2 T_{c}\right)}{\operatorname{ess} \inf } u \geq \vartheta_{c} .
$$

We begin the proof by showing the following reverse Hölder's inequality. It is a consequence of Caccioppoli estimate, Lemma 2.2, and a parabolic Sobolev's estimate. The technique is due to Moser and it was first time used in the elliptic case in [23].

Lemma 5.2. Suppose that $u \geq 1$ is a weak supersolution in an open set which compactly contains $B(0,2) \times\left(0,2^{p}\right)$. Then for every $p-2<q<p-1+p / n$, 
$s=p-2+(1+p / n)^{-M}(q-(p-2)), M=1,2, \ldots$, there is a constant $C=C\left(n, p, \mathcal{A}_{0}, \mathcal{A}_{1}, q, s\right)$ such that

$$
\left(\int_{0}^{2} \int_{B(0, \rho)} u^{q} d x d t\right)^{1 /(q-p+2)} \leq\left(\frac{C}{(2-\rho)^{n+p}} \int_{0}^{2^{p}} \int_{B(0,2)} u^{s} d x d t\right)^{1 /(s-p+2)}
$$

for all $1<\rho<2$.

Proof. Let $M \in \mathbb{N}$ and set

$$
R_{0}=2, \quad R_{j}=\left(2-(2-\rho) \frac{1-2^{-j}}{1-2^{-M}}\right), \quad j=0,1, \ldots, M .
$$

We also denote

$$
U_{j}=B\left(0, R_{j}\right) \times\left(0, R_{j}^{p}\right) .
$$

We choose test functions $\varphi_{j} \in C^{\infty}\left(U_{j}\right) \cap C\left(\bar{U}_{j}\right), j=0,1,2, \ldots, M$, such that $0 \leq \varphi_{j} \leq 1, \varphi_{j}=1$ in $U_{j+1}$, and $\varphi_{j}=0$ on $B_{j} \times\left\{R_{j}^{p}\right\}$ and on $\partial B_{j} \times\left(0, R_{j}^{p}\right)$. Moreover, there is a constant $C$ such that

$$
\left|\nabla \varphi_{j}\right| \leq \frac{C 2^{j}}{2-\rho}, \quad \frac{\partial \varphi_{j}^{p}}{\partial t} \geq-\frac{C 2^{p j}}{(2-\rho)^{p}},
$$

$j=0,1,2, \ldots, M$. We then apply parabolic Sobolev's inequality (see [8, Proposition 3.1, page 7]) and obtain

$$
\begin{aligned}
& \int_{U_{j+1}} u^{\kappa \alpha} d x d t \leq C \int_{U_{j}}\left(u^{\alpha / p} \varphi_{j}^{\beta / p}\right)^{\kappa p} d x d t \\
& \leq \int_{U_{j}}\left|\nabla\left(u^{\alpha / p} \varphi_{j}^{\beta / p}\right)\right|^{p} d x d t\left(\operatorname{ess} \sup _{0<t<R_{j}^{p}} \int_{B_{j}}\left(u^{\alpha / p} \varphi_{j}^{\beta / p}\right)^{(\kappa-1) n} d x\right)^{p / n}
\end{aligned}
$$

for some $\alpha \in \mathbb{R}, \beta \geq p$ and $\kappa>1$. The inequality above is formal in sense that we first calculate (5.2) for $\min (u, k)$ instead of $u$. We then use the monotone convergence theorem to pass to the limit as $k \rightarrow \infty$. We choose

$$
\alpha=p-1+\varepsilon, \quad \kappa=1+\frac{p(1+\varepsilon)}{n(p-1+\varepsilon)}, \quad \beta=\frac{p(p-1+\varepsilon)}{1+\varepsilon},
$$

where $-1<\varepsilon<0$. We use Lemma 2.2 to estimate terms on the right hand side of (5.2). First, we have

$$
\begin{aligned}
& \underset{0<t<R_{j}^{p}}{\operatorname{ess} \sup _{B_{j}}} \int_{0<t<R_{j}^{p}}\left(u^{\alpha / p} \varphi_{j}^{\beta / p}\right)^{(\kappa-1) n} d x=\operatorname{ess}_{0<t<\sup _{j}} u^{1+\varepsilon} \varphi_{j}^{p} d x \\
& \leq \frac{C}{|\varepsilon|^{p}(1+\varepsilon)}\left(\int_{U_{j}} u^{p-1+\varepsilon}\left|\nabla \varphi_{j}\right|^{p} d x d t+\int_{U_{j}} u^{1+\varepsilon}\left(-\frac{\partial \varphi_{j}^{p}}{\partial t}\right)_{+} d x d t\right) .
\end{aligned}
$$


Also

$$
\begin{aligned}
& \int_{U_{j}}\left|\nabla\left(u^{\alpha / p} \varphi_{j}^{\beta / p}\right)\right|^{p} d x d t \\
& \leq C\left(\int_{U_{j}}|\nabla u|^{p} u^{-1+\varepsilon} \varphi_{j}^{p} d x d t+\frac{1}{1+\varepsilon} \int_{U_{j}}\left|\nabla \varphi_{j}\right|^{p} u^{p-1+\varepsilon} d x d t\right) \\
& \leq \frac{C}{|\varepsilon|^{p}(1+\varepsilon)}\left(\int_{U_{j}}\left|\nabla \varphi_{j}\right|^{p} u^{p-1+\varepsilon} d x d t+\int_{U_{j}} u^{1+\varepsilon}\left(-\frac{\partial \varphi_{j}^{p}}{\partial t}\right)_{+} d x d t\right)
\end{aligned}
$$

holds. Consequently, we obtain

$$
\begin{aligned}
& \int_{U_{j+1}} u^{p-1+p / n+\gamma \varepsilon} d x d t \\
& \leq\left(\frac{C}{|\varepsilon|^{p}(1+\varepsilon)} \int_{U_{j}}\left(u^{p-1+\varepsilon}\left|\nabla \varphi_{j}\right|^{p}+u^{1+\varepsilon}\left(-\frac{\partial \varphi_{j}^{p}}{\partial t}\right)_{+}\right) d x d t\right)^{\gamma}
\end{aligned}
$$

where $\gamma=1+p / n$. Moreover, the assumption $u \geq 1$ implies

$$
\int_{U_{j+1}} u^{p-1+p / n+\gamma \varepsilon} d x d t \leq\left(\frac{C}{|\varepsilon|^{p}(1+\varepsilon)} \frac{2^{j p}}{(2-\rho)^{p}} \int_{U_{j}} u^{p-1+\varepsilon} d x d t\right)^{\gamma}
$$

We set

$$
\varepsilon_{j}=\gamma^{j}\left(\varepsilon_{0}+1\right)-1, \quad \alpha_{j}=p-1+\varepsilon_{j},
$$

where $-1<\varepsilon_{0}<-1+\gamma^{-M}, j=0,1, \ldots M$. This leads to the equality $p-1+$ $p / n+\gamma \varepsilon_{j}=p-1+\varepsilon_{j+1}$ and, hence, to the inequality

$$
\int_{U_{j+1}} u^{\alpha_{j+1}} d x d t \leq\left(\frac{1}{\left|\varepsilon_{j}\right|^{p}\left(1+\varepsilon_{j}\right)} \frac{C 2^{j p}}{(2-\rho)^{p}} \int_{U_{j}} u^{\alpha_{j}} d x d t\right)^{\gamma}
$$

for $j=0,1, \ldots, M$. We choose

$$
\varepsilon_{0}=-1+\gamma^{-1-M}(q-(p-2)), \quad \alpha_{0}=s, \quad \alpha_{M+1}=q .
$$

The choice implies the estimate

$$
\frac{1}{\left|\varepsilon_{j}\right|^{p}\left(1+\varepsilon_{j}\right)} \leq \frac{1}{\left|\varepsilon_{M}\right|^{p}\left(1+\varepsilon_{0}\right)}=\frac{\gamma^{p}}{(p-1+p / n-q)^{p}} \frac{1}{s-(p-2)} .
$$

We conclude that with the constant

$$
\widetilde{C}=\frac{1}{(p-1+p / n-q)(s-(p-2))^{1 / p}},
$$


we have

$$
\int_{U_{j+1}} u^{\alpha_{j+1}} d x d t \leq\left(\frac{C \widetilde{C}^{p} 2^{j p}}{(2-\rho)^{p}} \int_{U_{j}} u^{\alpha_{j}} d x d t\right)^{\gamma}
$$

We iterate this and obtain

$$
\int_{U_{M}} u^{q} d x d t \leq\left(\frac{C \widetilde{C}^{p}}{(2-\rho)^{p}}\right)^{\sum_{j=1}^{M+1} \gamma^{j}} \prod_{j=0}^{M-1} 2^{p(M-j) \gamma^{j+1}}\left(\int_{U_{0}} u^{s} d x d t\right)^{\gamma^{M+1}}
$$

Now, a direct calculation gives

$$
p \sum_{j=1}^{M+1} \gamma^{j}=\frac{p \gamma}{\gamma-1}\left(\gamma^{M+1}-1\right)=(n+p)\left(\gamma^{M+1}-1\right)
$$

and

$$
\prod_{j=0}^{M-1} 2^{p(M-j) \gamma^{j+1}}=\left(\prod_{j=1}^{M} 2^{p j \gamma^{-j}}\right)^{\gamma^{M+1}} \leq\left(2^{p \gamma /(\gamma-1)^{2}}\right)^{\gamma^{M+1}}
$$

Thus we arrive at

$$
\left(\int_{U_{M}} u^{q} d x d t\right)^{1 /(q-(p-2))} \leq\left(\frac{C \widetilde{C}^{n+p}}{(2-\rho)^{n+p}} \int_{U_{0}} u^{s} d x d t\right)^{1 /(s-(p-2))}
$$

which finishes the proof.

Using the lemma, we can show that under the assumption (5.1), $L^{q}$-integrals of supersolutions are uniformly bounded up to the critical exponent.

Lemma 5.3. Let $u$ be a non-negative weak supersolution in an open set which compactly contains $B(0,2) \times\left(0,2^{p}\right)$ such that $(5.1)$ holds. Then for all $p-2<q<$ $p-1+p / n$ there exists a constant $C=C\left(n, p, \mathcal{A}_{0}, \mathcal{A}_{1}, q\right)$ such that

$$
\int_{0}^{2} \int_{B(0,3 / 2)} u^{q} d x d t \leq C
$$

Proof. We define the supersolution $v=u+1$ and set

$$
\delta=\frac{\sigma}{2+2 \sigma}
$$

It follows from (5.1) that

$$
\int_{B(0,2)} v^{\delta}(x, t) d x \leq C
$$


for almost every $0<t<2^{p}$, where $C=C\left(n, p, \mathcal{A}_{0}, \mathcal{A}_{1}\right)$. We set

$$
U(s)=B(0, s) \times\left(0,2^{p}\right) .
$$

Let $\varphi \in C_{0}^{\infty}(B(0, S)), 0 \leq \varphi \leq 1, \varphi=1$ in $U(s)$ and $|\nabla \varphi| \leq C /(S-s)$, where $7 / 4 \leq s<S \leq 2$. We apply (5.2) with

$$
\alpha=p-2+\delta, \quad \kappa=1+\frac{p \delta}{n(p-2+\delta)}, \quad \beta=\frac{p(p-2+\delta)}{\delta}
$$

and arrive by (5.3) at

$$
\begin{aligned}
& \int_{U(s)} v^{p-2+\delta(1+p / n)} d x d t \\
& \leq C \int_{U(S)}\left|\nabla\left(v^{(p-2+\delta) / p} \varphi\right)\right|^{p} d x d t\left(\operatorname{ess~sup}_{0<t<2^{p}} \int_{B(0,2)} v^{\delta} d x\right)^{p / n} \\
& \leq C \int_{U(S)}\left|\nabla\left(v^{(p-2+\delta) / p} \varphi\right)\right|^{p} d x d t .
\end{aligned}
$$

We use (2.3) with $\varepsilon=-1+\delta$ and obtain again by (5.3) that

$$
\begin{aligned}
& \int_{U(S)}\left|\nabla\left(v^{(p-2+\delta) / p} \varphi\right)\right|^{p} d x d t \\
& \leq C \int_{U(S)}|\nabla \varphi|^{p} v^{p-2+\delta} d x d t+C \underset{0<t<2^{p}}{\operatorname{ess} \sup } \int_{B(0, S)} v^{\delta} d x \\
& \leq C \int_{U(S)}|\nabla \varphi|^{p} v^{p-2+\delta} d x d t+C .
\end{aligned}
$$

We then apply Young's inequality and get

$$
\begin{aligned}
& \int_{U(S)}|\nabla \varphi|^{p} v^{p-2+\delta} d x d t \\
& \leq \frac{1}{2 C} \int_{U(S)} v^{p-2+\delta(1+p / n)} d x d t+C\left(\frac{1}{S-s}\right)^{p+n(p-2+\delta) / \delta} .
\end{aligned}
$$

Therefore, we have

$$
\begin{aligned}
& \int_{U(s)} v^{p-2+\delta(1+p / n)} d x d t \\
& \leq \frac{1}{2} \int_{U(S)} v^{p-2+\delta(1+p / n)} d x d t+C\left(\frac{1}{S-s}\right)^{p+n(p-2+\delta) / \delta} .
\end{aligned}
$$

An iteration argument (see e.g. [15, Lemma 5.1]) shows that

$$
\int_{0}^{2^{p}} \int_{B(0,7 / 4)} v^{p-2+\delta(1+p / n)} d x d t \leq C .
$$

An application of Lemma 5.2 then completes the proof. 
Lemma 5.4. Let $u$ be as in Lemma 5.3. Then there exists a constant $C=$ $C\left(n, p, \mathcal{A}_{0}, \mathcal{A}_{1}\right)$ such that

$$
\int_{0}^{1} \int_{B(0,5 / 4)}|\nabla u|^{p-1} d x d t \leq C .
$$

Proof. Let $v=u+1$. We use Hölder's inequality and obtain

$$
\begin{aligned}
& \int_{0}^{1} \int_{B(0,5 / 4)}|\nabla u|^{p-1} d x d t \\
& =\int_{0}^{1} \int_{B(0,5 / 4)}|\nabla v|^{p-1} v^{-(1+\varepsilon)(p-1) / p} v^{(1+\varepsilon)(p-1) / p} d x d t \\
& \leq\left(\int_{0}^{1} \int_{B(0,5 / 4)}|\nabla v|^{p} v^{-1-\varepsilon} d x d t\right)^{(p-1) / p} \\
& \quad \times\left(\int_{0}^{1} \int_{B(0,5 / 4)} v^{p-1+\varepsilon(p-1)} d x d t\right)^{1 / p},
\end{aligned}
$$

where

$$
\varepsilon=\frac{p}{2 n(p-1)}<1
$$

By Lemma 5.3, we obtain

$$
\int_{0}^{1} \int_{B(0,1)} v^{p-1+\varepsilon(p-1)} d x d t \leq C
$$

Furthermore, we choose $\varphi \in C^{\infty}(B(0,3 / 2) \times(0,2)), 0 \leq \varphi \leq 1$, such that

$$
\varphi=0 \quad \text { on } \quad \partial B(0,3 / 2) \times(0,2) \cup B(0,3 / 2) \times\{2\}
$$

and

$$
\varphi=1 \quad \text { in } \quad B(0,5 / 4) \times(0,1), \quad|\nabla \varphi|+\left(-\frac{\partial \varphi}{\partial t}\right)_{+} \leq C .
$$

We obtain by Lemma 2.2 that

$$
\begin{aligned}
\int_{0}^{1} \int_{B(0,5 / 4)}|\nabla v|^{p} v^{-1-\varepsilon} d x d t \leq & C \int_{0}^{2} \int_{B(0,3 / 2)} v^{p-1-\varepsilon}|\nabla \varphi|^{p} d x d t \\
& +C \int_{0}^{2} \int_{B(0,3 / 2)} v^{1-\varepsilon}\left(-\frac{\partial \varphi^{p}}{\partial t}\right)_{+} d x d t .
\end{aligned}
$$

The result now follows by Lemma 5.3 and Hölder's inequality. 
Lemma 5.5. Under the hypothesis of Lemma 5.3 there is a constant $C=$ $C\left(n, p, \mathcal{A}_{0}, \mathcal{A}_{1}\right)$ such that if

$$
f_{B(0,1)} u(x, 0) d x \geq 2 C,
$$

then

$$
\underset{0<t<1}{\operatorname{essinf}} f_{B(0,5 / 4)} u(x, t) d x \geq C .
$$

Proof. We take $\varphi \in C_{0}^{\infty}(B(0,5 / 4)), 0 \leq \varphi \leq 1, \varphi=1$ in $B(0,1)$ and $|\nabla \varphi| \leq$ $C$. Since 0 is a Lebesgue instant and $u$ is a supersolution in an open set which compactly contains $B(0,2) \times(0,2)$, we may substitute $\varphi$ in (2.4) and obtain

$$
\begin{aligned}
f_{B(0,5 / 4)} u(x, T) \varphi(x) d x \geq & f_{B(0,5 / 4)} u(x, 0) \varphi(x) d x \\
& -\mathcal{A}_{1} \int_{0}^{1} f_{B(0,5 / 4)}|\nabla u|^{p-1}|\nabla \varphi| d x d t
\end{aligned}
$$

for almost every $0<T<1$. The result then follows by Lemma 5.4.

We also need the following simple tool.

Lemma 5.6. Let $\Omega$ be a bounded domain in $\mathbb{R}^{n}$. Suppose that $f$ is a measurable function in $\Omega$ satisfying

$$
f_{\Omega} f d x \geq 2 N
$$

and

$$
\left(f_{\Omega} f^{q} d x\right)^{1 / q} \leq C N
$$

for some $1<q \leq+\infty$ and $C \geq 2$, then

$$
|\{x \in \Omega: f(x)>N\}| \geq C^{-q /(q-1)}|\Omega| .
$$

Proof. By Hölder's inequality we have

$$
\begin{aligned}
2 N & \leq f_{\Omega} f d x=\frac{1}{|\Omega|}\left(\int_{\{f>N\}} f d x+\int_{\{f \leq N\}} f d x\right) \\
& \leq C N\left(\frac{1}{|\Omega|}|\{f>N\}|\right)^{1-1 / q}+N,
\end{aligned}
$$

which implies the result. 
Proof of Lemma 5.1. We obtain from Lemmas 5.3, 5.5 and 5.6 that we find positive constants $C_{i}=C_{i}\left(n, p, \mathcal{A}_{0}, \mathcal{A}_{1}\right), i=1,2$, such that

$$
\left|\left\{(x, t) \in Q: u(x, t) \geq \frac{1}{C_{1}}\right\}\right| \geq \frac{1}{C_{2}}|Q|,
$$

where $Q=B(0,5 / 4) \times(0,1)$, provided that $M_{c}$ is big enough. Note that $M_{c}$ can be chosen to depend only on $n, p, \mathcal{A}_{0}$, and $\mathcal{A}_{1}$. Consequently, there is a Lebesgue instant $0<\bar{t}<1$ such that

$$
\left|\left\{x \in B(0,5 / 4): u(x, \bar{t}) \geq \frac{1}{C_{1}}\right\}\right| \geq \frac{1}{C_{2}}|B(0,5 / 4)| .
$$

The assertion follows now by the expansion of positivity, Proposition 3.1.

\section{Proofs of main results}

We now proceed to the proofs of our main results.

Proof of Theorem 1.1. Let $t_{0}<t_{1}<t_{0}+T_{0}$ be a Lebesgue instant of $u$. For the brevity, we denote

$$
N=f_{B\left(x_{0}, R_{0}\right)} u\left(x, t_{1}\right) d x
$$

and assume that $N>0$. We introduce a scaled function

$$
v(x, t)=\frac{M_{c}}{N} u\left(x_{0}+R_{0} x, t_{1}+\left(M_{c} / N\right)^{p-2} R_{0}^{p} t\right),
$$

where $M_{c}$ is as in Lemma 5.1. The function $v$ is a supersolution in $B(0,8) \times$ $\left(0,\left(N / M_{c}\right)^{p-2}\left(T_{0}+t_{0}-t_{1}\right) / R_{0}^{p}\right)$ and

$$
f_{B(0,1)} v(x, 0) d x=M_{c} .
$$

Let $T_{h}, \vartheta_{h}$ and $T_{c}, \vartheta_{c}$ be as in Lemma 4.1 and 5.1, respectively. Suppose then that

$$
\left(M_{c} / N\right)^{p-2}\left(T_{0}+t_{0}-t_{1}\right) / R_{0}^{p} \leq 2 T^{*},
$$

for some $T^{*} \geq \max \left\{T_{h}, T_{c}\right\}$, or equivalently,

$$
N \geq\left(\frac{C_{1} R_{0}^{p}}{T_{0}+t_{0}-t_{1}}\right)^{1 /(p-2)}
$$

where $C_{1}=2 T^{*} M_{c}^{p-2}$. Then either Lemma 4.1 or Lemma 5.1 holds and, hence,

$$
\underset{B(0,2) \times\left(T_{h}, 2 T_{h}\right)}{\operatorname{ess} \inf } v \geq \vartheta_{h} \quad \text { or } \quad \operatorname{ess~inf}_{B(0,2) \times\left(T_{c}, 2 T_{c}\right)} v \geq \vartheta_{c} .
$$


In either case we may again apply Proposition 3.1 and obtain that there are constants $\vartheta$ and $T^{*}$ depending only on $n, p, \mathcal{A}_{0}$, and $\mathcal{A}_{1}$ such that

$$
\underset{B(0,2) \times\left(T^{*}, 2 T^{*}\right)}{\operatorname{essinf}} v \geq \vartheta .
$$

We then scale back and obtain the desired result.

We use the local Harnack estimate to prove the second main result, the global Harnack estimate.

Proof of Theorem 1.2. First, we scale the supersolution as

$$
v(x, t)=u\left(x_{0}+(R / M) x,(R / M)^{p} t\right),
$$

where $0<M \leq 1$. Then $v$ is a supersolution in

$$
\mathbb{R}^{n} \times\left(0, T_{0}(M / R)^{p}\right) .
$$

We have

$$
f_{B\left(x_{0}, R\right)} u\left(x, t_{0}\right) d x=f_{B(0, M)} v\left(x, t_{1}\right) d x \leq M^{-n} f_{B(0,1)} v\left(x, t_{1}\right) d x,
$$

where $t_{1}=(M / R)^{p} t_{0}$. Let $C_{1}$ and $C_{2}$ be as in Theorem 1.1 and suppose that

$$
f_{B(0,1)} v\left(x, t_{1}\right) d x>2\left(\frac{C_{1}}{\left(T_{0}-t_{0}\right)(M / R)^{p}}\right)^{1 /(p-2)} .
$$

We then apply Theorem 1.1 and obtain

$$
f_{B(0,1)} v\left(x, t_{1}\right) d x \leq\left(\frac{C_{1}}{\left(T_{0}-t_{0}\right)(M / R)^{p}}\right)^{1 /(p-2)}+C_{2} \underset{Q_{1}}{\operatorname{essinf} v}
$$

where $Q_{1}=B(0,4) \times\left(t_{1}+T_{1} / 2, t_{1}+T_{1}\right)$ and

$$
T_{1}=C_{1}\left(\frac{1}{2} f_{B(0,1)} v\left(x, t_{1}\right) d x\right)^{2-p}<\left(T_{0}-t_{0}\right)(M / R)^{p}
$$

by the condition (6.2). It follows that

$$
f_{B(0,1)} v\left(x, t_{1}\right) d x \leq \underset{Q_{1}}{2 C_{2}} \underset{Q_{1}}{\operatorname{essinf}} v .
$$

Furthermore, we choose

$$
M^{p}=\frac{C_{1} R^{p}}{T}\left(\frac{1}{2} f_{B(0,1)} v\left(x, t_{1}\right) d x\right)^{2-p},
$$


so that

$$
T=(R / M)^{p} C_{1}\left(\frac{1}{2} f_{B(0,1)} v\left(x, t_{1}\right) d x\right)^{2-p}<T_{0}-t_{0} .
$$

The choice of $M$ leads by (6.1) to the inequality

$$
M^{\lambda} \leq \frac{C_{1} R^{p}}{T}\left(\frac{1}{2} f_{B\left(x_{0}, R\right)} u\left(x, t_{0}\right) d x\right)^{2-p},
$$

where $\lambda=n(p-2)+p$. Thus the requirement $M \leq 1$ is certainly fulfilled if

$$
f_{B\left(x_{0}, R\right)} u\left(x, t_{0}\right) d x \geq\left(\frac{2^{p-2} C_{1} R^{p}}{T}\right)^{1 /(p-2)} .
$$

For such initial masses we have by (6.3) that

$$
M^{n} f_{B\left(x_{0}, R\right)} u\left(x, t_{0}\right) d x \leq 2 C_{2} \underset{Q_{2}}{\operatorname{essinf}} u
$$

where $Q_{2}=B\left(x_{0}, 4 R / M\right) \times\left(t_{0}+T / 2, t_{0}+T\right)$. Note that

$$
\underset{Q_{1}}{\operatorname{essinf}} v=\underset{Q_{2}}{\operatorname{ess} \inf u}
$$

by the definition of $M$. Moreover, we obtain, again by (6.3), that

$$
\begin{aligned}
M^{-n} & =C\left(\frac{T}{R^{p}}\right)^{n / p}\left(\int_{B(0,1)} v\left(x, t_{1}\right) d x\right)^{n(p-2) / p} \\
& \leq C\left(\frac{T}{R^{p}}\right)^{n / p} \underset{Q_{2}}{\operatorname{essinf}} u^{n(p-2) / p} .
\end{aligned}
$$

We combine the estimates and conclude that

$$
f_{B\left(x_{0}, R\right)} u\left(x, t_{0}\right) d x \leq C\left(\frac{T}{R^{p}}\right)^{n / p} \underset{Q_{2}}{\operatorname{essinf}} u^{\lambda / p} .
$$

Recall that $Q \subset Q_{2}$, where $Q=B\left(x_{0}, 4 R\right) \times\left(t_{0}+T / 2, t_{0}+T\right)$, since $M \leq 1$. This proves the result.

The local intrinsic Harnack inequality follows now for weak solutions. First, similar arguments that were used to prove Lemma 5.2, can be applied for subsolutions. As a result we have the following theorem. In [8, Theorem 4.1, page 122] the same result is obtained by using De Giorgi's iteration method. 
Theorem 6.1. Let $u$ be a non-negative subsolution in an open set which compactly contains $B\left(x_{0}, R\right) \times\left(t_{0}-T, t_{0}\right)$. Then there exists a constant $C=C\left(n, p, \mathcal{A}_{0}, \mathcal{A}_{1}\right)$ such that

$$
\underset{Q}{\operatorname{ess} \sup } u \leq C\left(\frac{R^{p}}{T}\right)^{1 /(p-2)}+C \frac{T}{R^{p}}\left(\operatorname{ess~sup}_{t_{0}-T<t<t_{0}} f_{B\left(x_{0}, R\right)} u d x\right)^{p-1},
$$

where $Q=B\left(x_{0}, R / 2\right) \times\left(t_{0}-T / 2, t_{0}\right)$.

The proof of intrinsic Harnack estimate is based on scaling and the following result. We emphasize the different roles of weak sub- and supersolutions.

Lemma 6.2. There is a constants $T^{*}$ and $\vartheta$ depending only on $n, p, \mathcal{A}_{0}$, and $\mathcal{A}_{1}$, such that if $u$ is a local non-negative weak solution to $(2.2)$ in $B(0,4) \times\left(-T^{*}, T^{*}\right)$ and

$$
\underset{1 / 4) \times(-1 / 4,0)}{\text { ess sup }} u \geq 1,
$$

then

$$
\underset{B(0,1) \times\left(T^{*} / 2, T^{*}\right)}{\operatorname{essinf}} u \geq \vartheta .
$$

Moreover, the constants $T^{*}$ and $\vartheta$ are stable as $p \rightarrow 2$.

Proof. Basic ingredients of the proof are Theorem 1.1 and Theorem 6.1. We first use Theorem 6.1 for subsolutions and get

$$
1 \leq C_{1}\left(\frac{R_{1}^{p}}{T_{1}}\right)^{1 /(p-2)}+C_{1} \frac{T_{1}}{R_{1}^{p}}\left(\operatorname{ess~sup}_{-T_{1}<t<0} f_{B\left(0, R_{1}\right)} u d x\right)^{p-1}
$$

with

$$
R_{1}=\frac{1}{2}, \quad \frac{R_{1}^{p}}{T_{1}}=\left(2 C_{1}\right)^{2-p}, \quad 1<T_{1}<T^{*} / 4 .
$$

We obtain for some Lebesgue instant $-T_{1}<t_{1}<0$ such that

$$
f_{B(0,1 / 2)} u\left(x, t_{1}\right) d x \geq \frac{2}{C_{2}} .
$$

We then apply Theorem 1.1 for supersolutions with $R_{0}=1 / 2, t_{0}=t_{1}-T^{*} / 4$, and $T_{0}=T^{*} / 4$ and obtain

$$
f_{B(0,1 / 2)} u\left(x, t_{1}\right) d x \leq\left(\frac{C_{3}}{2^{p}\left(T^{*} / 4-t_{1}\right)}\right)^{1 /(p-2)}+\underset{Q}{C_{4} \operatorname{essinf} u,}
$$

where $Q=B(0,2) \times\left(t_{1}+T / 2, t_{1}+T\right)$ and

$$
T=\min \left\{T^{*} / 4-t_{1}, C_{3} 2^{-p}\left(f_{B(0,1 / 2)} u\left(x, t_{1}\right) d x\right)^{2-p}\right\} .
$$


The choice $T^{*}=2^{2-p} C_{3} C_{2}^{p-2}$ leads to

$$
\underset{Q}{\operatorname{essinf}} u \geq \frac{1}{C_{5}}
$$

We may now apply Proposition 3.1 on the expansion of positivity and find an instant $T^{*}$ and level $\vartheta$ as in the statement. This finishes the proof.

We now define the values of $u$ pointwise via

$$
u(x, t)=\lim _{R \rightarrow 0} \operatorname{ess~sup}_{B(x, R) \times\left(t, t-R^{p}\right)} u .
$$

We then obtain the local Harnack estimate.

Theorem 6.3. Suppose that $u$ is a non-negative weak solution in $Q_{1}=B\left(x_{1}, R_{1}\right) \times$ $\left(t_{1}, t_{1}+T_{1}\right)$. Then there exist constants $C_{i}=C_{i}\left(n, p, \mathcal{A}_{0}, \mathcal{A}_{1}\right), i=1,2$, such that if

$$
Q_{0}=B\left(x_{0}, 4 R\right) \times\left(t_{0}-C_{1} u\left(x_{0}, t_{0}\right)^{2-p} R^{p}, t_{0}+C_{1} u\left(x_{0}, t_{0}\right)^{2-p} R^{p}\right)
$$

belongs to $Q_{1}$ for $R>0$, then

$$
u\left(x_{0}, t_{0}\right) \leq C_{2} \underset{Q}{\operatorname{ess} \inf } u
$$

where

$$
Q=B\left(x_{0}, R\right) \times\left(t_{0}+C_{1} u\left(x_{0}, t_{0}\right)^{2-p} R^{p} / 2, t_{0}+C_{1} u\left(x_{0}, t_{0}\right)^{2-p} R^{p}\right) .
$$

The constants $C_{1}$ and $C_{2}$ are stable as $p \rightarrow 2$.

Proof. We assume that $u\left(x_{0}, t_{0}\right)>0$. Let $C_{1}=T^{*}$, where $T^{*}$ is as in Lemma 6.2. Then the scaled solution

$$
v(x, t)=\frac{1}{u\left(x_{0}, t_{0}\right)} u\left(x_{0}+x / R, t_{0}+u\left(x_{0}, t_{0}\right)^{2-p} t / R^{p}\right)
$$

satisfy the assumptions of Lemma 6.2, and by scaling back we get the result.

\section{References}

[1] E. ACERBi and G. MingIOnE, Gradient estimates for a class of parabolic systems, Duke Math. J. 136 (2) (2007), 285-320.

[2] D. G. Aronson and L. A. CAFfarelli, The initial trace of a solution of the porous medium equation, Trans. Amer. Math. Soc. 280 (1) (1983), 351-366.

[3] D. G. ARONSON and J. SERRIN, Local behavior of solutions of quasilinear parabolic equations, Arch. Ration. Mech. Anal. 25 (1967), 81-122. 
[4] H. J. CHOE and J. H. LEE, Cauchy problem for nonlinear parabolic equations, Hokkaido Math. J. 27 (1) (1998), 51-75.

[5] B. E. J. DAhlberg and C. E. Kenig, Non-Negative solutions of the porous medium equation, Comm. Partial Differential Equations 9 (5) (1984), 409-437.

[6] P. Daskalopoulos and C. E. Kenig, "Degenerate Diffusions", EMS Tracts in Mathematics, Vol. 1, European Mathematical Society (EMS), Zürich, 2007, Initial value problems and local regularity theory.

[7] E. De GIORGI, Sulla differenziabilità e l'analiticità delle estremali degli integrali multipli regolari, Mem. Accad. Sci. Torino. Cl. Sci. Fis. Mat. Natur. 3 (3) (1957), 25-43.

[8] E. DiBenedetto, "Degenerate Parabolic Equations", Universitext, Springer-Verlag, New York, 1993.

[9] E. DiBenedetto, U. GianazZa and V. Vespri, Intrinsic Harnack estimates for nonnegative local solutions of degenerate parabolic equations, Acta Math. 200 (2008), 181209.

[10] E. DiBenedetto, U. GianazZa and V. Vespri, Subpotential lower bounds for nonnegative solutions to certain quasi-linear degenerate parabolic equations, Duke Math. J. 143 (2008), 1-15.

[11] E. DiBenedetto and M. A. Herrero, On the Cauchy problem and initial traces for a degenerate parabolic equation, Trans. Amer. Math. Soc. 314 (1) (1989), 187-224.

[12] E. DiBenedetto, J. M. Urbano and V. Vespri, Current issues on singular and degenerate evolution equations, In: "Evolutionary Equations", Vol. I, Handb. Differ. Equ., 169-286, North-Holland, Amsterdam, 2004.

[13] E. DiBenedetto and N. S. TRUdinger, Harnack inequalities for quasiminima of variational integrals, Ann. Inst. H. Poincaré Anal. Non Linéaire 1 (1984), 295-308, MR MR778976 (86g:49007).

[14] U. GIANAZZA and V. VESPRI, A Harnack inequality for a degenerate parabolic equation, J. Evol. Equ. 6 (2) (2006), 247-267.

[15] M. Giaquinta, "Introduction to Regularity Theory for Nonlinear Elliptic Systems", Lectures in Mathematics ETH Zürich, Birkhäuser Verlag, Basel, 1993.

[16] J. Heinonen, "Lectures on Analysis on Metric Spaces", Universitext, Springer-Verlag, New York, 2001.

[17] J. Heinonen, T. Kilpeläinen and O. Martio, "Nonlinear Potential Theory of Degenerate Elliptic Equations", Oxford Mathematical Monographs, The Clarendon Press Oxford University Press, New York, 1993, Oxford Science Publications.

[18] A. V. IVANOV, The Harnack inequality for generalized solutions of second order quasilinear parabolic equations, Trudy Mat. Inst. Steklov. 102 (1967), 51-84.

[19] J. Kinnunen and N. Shanmugalingam, Regularity of quasi-minimizers on metric spaces, Manuscripta Math. 105 (3) (2001), 401-423.

[20] N. V. KRYLOV and M. V. SAFONOV, A certain property of solutions of parabolic equations with measurable coefficients, Math. USSR Izv. 16 (1) (1981), 151-164.

[21] M. Kurihara, On a Harnack inequality for nonlinear parabolic equations, Publ. Res. Inst. Math. Sci. 3 (1967/1968), 211-241.

[22] J.-L. Lions, "Quelques Méthodes de Résolution des Problèmes aux Limites non Linéaires", Dunod, 1969.

[23] J. Moser, On Harnack's theorem for elliptic differential equations, Comm. Pure Appl. Math. 14 (1961), 577-591.

[24] J. Moser, A Harnack inequality for parabolic differential equations, Comm. Pure Appl. Math. 17 (1964), 101-134.

[25] J. Moser, Correction to A Harnack inequality for parabolic differential equations, Comm. Pure Appl. Math. 20 (1967), 231-236.

[26] J. Moser, On a pointwise estimate for parabolic differential equations, Comm. Pure Appl. Math. 24 (1971), 727-740. 
[27] N. S. Trudinger, Pointwise estimates and quasilinear parabolic equations, Comm. Pure Appl. Math. 21 (1968), 205-226.

[28] J. M. URBAnO, "The Method of Intrinsic Scaling. a Systematic Approach to Regularity for Degenerate and Singular PDEs", Lecture Notes in Mathematics, Vol. 1930, Springer, 2008.

[29] J. L. VAZQUEZ, "The Porous Medium Equation Mathematical Theory", Oxford Mathematical Monographs, Clarendon Press, 2006.

[30] J. L. VAZQUEZ, "Smoothing and Decay Estimates for Nonlinear Diffusion Equations Equations of Porous Medium Type", Vol. 33 of Oxford Lecture Series in Mathematics and Its Applications, Oxford University Press, 2006.

Helsinki University of Technology Institute of Mathematics

P.O. Box 1100

FI-02015 TKK, Finland

tuomo.kuusi@tkk.fi 